\title{
Statistical Properties and Applications of the Exponentiated Chen-G Family of Distributions: Exponential Distribution as a Baseline Distribution
}

\author{
Phillip Oluwatobi Awodutire \\ University of Africa, Toru Orua
}

\begin{abstract}
In this work, the Exponentiated Chen-G family of distributions is studied by generalizing the Chen-G family of distributions through the introduction of an additional shape parameter. The mixture properties of the derived family are studied. Some statistical properties of the family were considered, including moments, entropies, moment generating function, order statistics, quantile function. The estimation of the parameters of the family of distributions was done using the maximum likelihood estimation method, considering complete and censored situations. Using the Exponential distribution as a baseline, the Exponentiated Chen Exponential distribution was obtained and its statistical properties were studied. The Exponentiated Chen Exponential distribution has the Exponentiated Exponential, Exponential, Chen Exponential distributions as submodels. Lastly, the Exponentiated Chen Exponential distribution was applied to two real data sets and the results were compared with its submodels and relative distributions.
\end{abstract}

Keywords: Chen-G family, exponential distribution, statistical properties, baseline distribution, maximum likelihood estimation.

\section{Introduction}

Many generalized families of distributions have been proposed and studied over the last two decades for modeling data in various applied areas such as economics, engineering, biological studies, environmental sciences, medical sciences, and finance. So, several classes of distributions have been constructed by extending common families of continuous distributions. These generalized distributions give more flexibility by adding one (or more) shape parameters to the baseline model. They were pioneered by Gupta, Gupta, and Gupta (1998) who proposed the Exponentiated-H class, which consists of raising the cumulative distribution function (cdf) to a positive power parameter. For $\mathrm{x}>0$, the Exponentiated-H family of distributions has the pdf

$$
f(x ; \beta)=\gamma j(x ; \beta) J(x ; \beta)^{\gamma-1}
$$

and the corresponding cdf as

$$
F(x ; \beta)=J(x ; \beta)^{\gamma}
$$


where $\gamma>0, \mathrm{j}(\mathrm{x} ; \beta)$ and $\mathrm{J}(\mathrm{x} ; \beta)$ are the baseline pdf and cdf respectively with parameter $\beta$. The Exponentiated-H has been extensively used in generalizing some baseline distributions. These include Exponentiated Weibull (Mudholkar and Srivastava 1993), Exponentiated Pareto (Ibrahim and Abu-Zinadah 2009), Exponentiated Generalized Lindley (Rodrigues, Percontini, and Hamedani 2017) e.t.c.

Furthermore, several new classes of distributions can be developed by adding one or more parameters to an existing distribution or family of distributions. In literature, many families of distributions have been proposed and extensively studied. Examples include Weibull-G (Bourguignon, Silva, and M. 2014), Transmuted-G (Shaw and Buckley 2009), Beta-G (Eugene, Lee, and Famoye 2002), Kumaraswamy-G (Nofal, Altun, Afify, and Ahsanullah 2019), Marshall-Olkin (Yousof, Afify, Nadarajah, Hamedani, and Aryal 2018), Logistic-X (Tahir, Cordeiro, Alzaatreh, Mansoor, and Zubair 2016), Chen-G (Anzagra, Sarpong, and Nasiru 2020), Lomax-G (Cordeiro, Ortega, Popović, and Pescim 2014) e.t.c.

These families of distributions have been further extended by the introduction of additional shape parameter(s). Some of these extended families of distributions include Kumaraswamy Odd Log-logistic family (Alizadeh, Emadi, Doostparast, Cordeiro, Ortega, and Pescim 2015), Kumaraswamy Marshall-Olkin family (Handique, Chakraborty, and Hamedani 2017), BetaWeibull-G (Yousof, Rasekhi, Afify, Ghosh, Alizadeh, and Hamedani 2017), Exponentiated Weibull-G Cordeiro, Afify, Yousof, Pescim, and Aryal (2017), Beta Transmuted-H (Afify, Yousof, and Nadarajah 2017), Kumaraswamy Transmuted-G (Afify, Cordeiro, Yousof, Alzaatreh, and Nofal 2016), Transmuted Weibull G (Alizadeh, Rasekhi, Yousof, and Hamedani 2017), Transmuted Exponentiated G (Yousof, Afify, Alizadeh, Butt, Hamedani, and Ali 2015), Transmuted Odd Frechet G (Badr, Elbatal, Jamal, Chesneau, and Elgarhy 2020) e.t.c.

In this work, a new class of distributions called the Exponentiated Chen-G distribution is proposed, which is obtained by mixing Exponentiated $\mathrm{H}$ family of distributions and the Chen- $\mathrm{G}$ family of distributions. From the Chen-G distribution, the Chen-Pareto (Awodutire 2020) was derived. The generalized distribution can accommodate different forms of the hazard function and contain few distributions as some special sub-models in literature.

Therefore, the plan of this work is as follows: Section 2, discusses the derivation of the new family of distributions with its subfamilies. Section 3 treats the linear representation of the distribution. In Section 4, the statistical properties of the distribution which includes moments, moment generating function, reliability functions, order statistics, entropy are studied. The estimation of parameters of the family of distributions under complete and right-censored observation was considered in section 5 using the maximum likelihood estimation method. Section 6 discusses the Exponentiated Chen Exponential distribution including its statistical properties, simulation studies, and applications to both complete and censored observations. Necessary conclusions were done in section 7 .

\section{The exponentiated Chen G family of distributions}

Consider a baseline cumulative distribution function (cdf) $\mathrm{G}(\mathrm{x} ; \beta)$ with corresponding probability density function (pdf) $\mathrm{g}(\mathrm{x} ; \beta)$ and parameter vector $\beta$. Then, the cdf of the Chen-G family of distributions (for $x>0$ ) is

$$
J(x ; \beta)=A\left[1-e^{\tau\left(1-e^{G(x ; \beta)^{\theta}}\right)}\right]
$$

with the corresponding pdf as

$$
j(x ; \beta)=A \tau \theta g(x ; \beta) G(x ; \beta)^{\theta-1} e^{G(x ; \beta)^{\theta}} e^{\tau\left(1-e^{G(x ; \beta)^{\theta}}\right)}
$$

where

$$
A=\frac{1}{1-e^{\tau(1-e)}}
$$

and $\mathrm{x}>0, \tau, \theta>0$.It is important to note that $\mathrm{A}$ is a normalizing constant, $\tau$ is the scale parameter and $\theta$ is the shape parameter. 
Inserting equation (4) in (1), the pdf $\mathrm{f}(\mathrm{x} ; \beta)$ of the Exponentiated Chen $\mathrm{G}$ family of distributions is obtained as

$$
f(x ; \beta)=\gamma A \tau \theta g(x ; \beta) G(x ; \beta)^{\theta-1} e^{G(x ; \beta)^{\theta}} e^{\tau\left(1-e^{G(x ; \beta)^{\theta}}\right)}\left(A\left[1-e^{\tau\left(1-e^{G(x ; \beta)^{\theta}}\right)}\right]\right)^{\gamma-1}
$$

which equivalently is

$$
f(x ; \beta)=\gamma A^{\gamma} \tau \theta g(x ; \beta) G(x ; \beta)^{\theta-1} e^{G(x ; \beta)^{\theta}} e^{\tau\left(1-e^{G(x ; \beta)^{\theta}}\right)}\left[1-e^{\tau\left(1-e^{G(x ; \beta)^{\theta}}\right)}\right]^{\gamma-1}
$$

and inserting (3) in (2), the cdf of the ECG distribution is

$$
F(x ; \beta)=\left(A\left[1-e^{\tau\left(1-e^{G(x ; \beta)^{\theta}}\right)}\right]\right)^{\gamma} .
$$

When $\gamma=1,(6)$ and (7) become the pdf and cdf the Chen-G family of distributions respectively (Anzagra et al. 2020). When $\tau=1$ and $\theta=1$, it gives the Exponentiated-G family of distributions (Gupta et al. 1998).

\section{Mixture representation}

In this section, the mixture representation of the pdf and cdf of the Exponentiated Chen$\mathrm{G}$ family of distributions is discussed. This linear representation is very important for the derivation of statistical properties of the family of distributions. It allows the properties to be expressed as mixtures.

From the pdf of the ECG family as

$$
f(x)=\gamma A^{\gamma} \tau \theta g(x) G(x)^{\theta-1} e^{G(x)^{\theta}} e^{\tau\left(1-e^{G(x)^{\theta}}\right)}\left[1-e^{\tau\left(1-e^{G(x)^{\theta}}\right)}\right]^{\gamma-1} .
$$

Consider the power series

$$
(1-q)^{r-1}=\sum_{y=0}^{\infty}(-1)^{r}\left(\begin{array}{c}
r-1 \\
y
\end{array}\right) q^{r}
$$

where $|q|<1$ and $\mathrm{r}>0$, then the equation

$$
\left[1-e^{\tau\left(1-e^{G(x ; \beta)^{\theta}}\right)}\right]^{\gamma-1}=\sum_{j=0}^{\infty}(-1)^{j}\left(\begin{array}{c}
\gamma-1 \\
j
\end{array}\right) e^{j \tau\left(1-e^{G(x ; \beta)^{\theta}}\right)} .
$$

Replacing equation (9) in (8), to have

$$
f(x ; \beta)=\gamma A^{\gamma} \tau \theta g(x ; \beta) G(x ; \beta)^{\theta-1} e^{G(x ; \beta)^{\theta}} \sum_{j=0}^{\gamma-1}(-1)^{j}\left(\begin{array}{c}
\gamma-1 \\
j
\end{array}\right) e^{(j+1) \tau\left(1-e^{G(x ; \beta)^{\theta}}\right)} .
$$

Furthermore, from the expression

$$
e^{x}=\sum_{k=0}^{\infty} \frac{x^{k}}{k !}
$$

then

$$
e^{(j+1) \tau\left(1-e^{G(x ; \beta)^{\theta}}\right)}=\sum_{k=0}^{\infty} \frac{(j+1)^{k} \tau^{k}\left(1-e^{G(x ; \beta)^{\theta}}\right)^{k}}{k !} .
$$

Inserting (11) in (10) gives

$$
f(x ; \beta)=\gamma A^{\gamma} \tau \theta g(x ; \beta) G(x ; \beta)^{\theta-1} e^{G(x ; \beta)^{\theta}}=\sum_{j=0}^{\infty} \sum_{k=0}^{\infty}(-1)^{j}\left(\begin{array}{c}
\gamma-1 \\
j
\end{array}\right) \frac{(j+1)^{k} \tau^{k}\left(1-e^{G(x ; \beta)^{\theta}}\right)^{k}}{k !} .
$$


Analyzing

$$
\left(1-e^{G(x ; \beta)^{\theta}}\right)^{k}=\sum_{l=0}^{k}(-1)^{l}\left(\begin{array}{l}
k \\
l
\end{array}\right) e^{l G(x ; \beta)^{\theta}}
$$

Inserting (13) in (12)

$$
f(x ; \beta)=\gamma A^{\gamma} \tau \theta g(x ; \beta) G(x ; \beta)^{\theta-1}=\sum_{j=0}^{\infty} \sum_{k=0}^{\infty} \sum_{l=0}^{k}(-1)^{j+l}\left(\begin{array}{c}
\gamma-1 \\
j
\end{array}\right)\left(\begin{array}{c}
k \\
l
\end{array}\right) \frac{(j+1)^{k} \tau^{k}}{k !} e^{(l+1) G(x ; \beta)^{\theta}} .
$$

Furthermore,

$$
e^{(l+1) G(x ; \beta)^{\theta}}=\sum_{m=0}^{\infty} \frac{(l+1)^{m} G(x ; \beta)^{m \theta}}{m !} .
$$

Inputing (15) in (14), it gives

$$
\begin{aligned}
f(x ; \beta) & =\gamma A^{\gamma} \tau \theta g(x ; \beta) \\
& =\sum_{j=0}^{\infty} \sum_{k=0}^{\infty} \sum_{l=0}^{k} \sum_{m=0}^{\infty}-1^{j+l}\left(\begin{array}{c}
\gamma-1 \\
j
\end{array}\right)\left(\begin{array}{c}
k \\
l
\end{array}\right) \frac{(j+1)^{k} \tau^{k}(l+1)^{m}}{k ! m !} G(x ; \beta)^{\theta(m+1)-1} .
\end{aligned}
$$

Rearranging gives

$f(x ; \beta)=\sum_{j=0}^{\infty} \sum_{k=0}^{\infty} \sum_{l=0}^{k} \sum_{m=0}^{\infty}(-1)^{j+l}\left(\begin{array}{c}\gamma-1 \\ j\end{array}\right)\left(\begin{array}{c}k \\ l\end{array}\right) \frac{(j+1)^{k} \tau^{k}(l+1)^{m}}{k ! m !(m+1)} \gamma A^{\gamma} \tau \theta(m+1) g(x ; \beta) G(x ; \beta)^{\theta(m+1)-1}$

to have

$$
f(x ; \beta)=\sum_{j=0}^{\infty} \sum_{k=0}^{\infty} \sum_{l=0}^{k} \sum_{m=0}^{\infty} t_{h} \Phi_{\theta(m+1)}
$$

where

$$
t_{h}=(-1)^{j+l}\left(\begin{array}{c}
\gamma-1 \\
j
\end{array}\right)\left(\begin{array}{c}
k \\
l
\end{array}\right) \frac{(j+1)^{k} \tau^{k}(l+1)^{m}}{k ! m !(m+1)} \gamma A^{\gamma} \tau
$$

and

$$
\Phi_{\theta(m+1)}=\theta(m+1) g(x ; \beta) G(x ; \beta)^{\theta(m+1)-1} .
$$

The expansion of the pdf of ECG distribution has therefore shown that the ECG pdf is an infinite combination of the Exp-G pdf. Integrating equation (18) gives mixture form of the cdf of the ECG family of distributions

$$
F(x ; \beta)=\sum_{j=0}^{\infty} \sum_{k=0}^{\infty} \sum_{l=0}^{k} \sum_{m=0}^{\infty} t_{h} \lambda_{\theta(m+1)}
$$

where

$$
\lambda_{\theta(m+1)}=G(x ; \beta)^{\theta(m+1)}
$$

is the cdf of the Exp-G distribution with power parameter $\theta(m+1)$. Therefore, it follows that the ECG density function is a linear combination of the Exp-G density functions which in turn infer that statistical properties of ECG distribution can be obtained from those of the Exp-G distribution.

\section{Statistical properties}

In this section, the statistical properties of the ECG distribution are comprehensively studied. The formulae derived throughout the paper can be easily handled in most symbolic computation software platforms such as Maple, Mathematica, and Matlab because of their ability to 
deal with analytic expressions of formidable size and complexity. Established explicit expressions to calculate statistical measures can be more efficient than computing them directly by numerical integration. The properties considered are moments, moment generating function, Renyi entropy, order statistics, survival function, and the hazard function.

\subsection{Moments}

In studying probability distributions, several features can be studied through their moments. Moments are often used to obtain important features of distributions such as measures of central tendency, dispersion, skewness, and kurtosis. In this section, the moments ECG family of distributions is considered.

The $r^{t h}$ moment of the ECG family of distributions can be obtained as:

$$
\begin{gathered}
E\left[X^{r}\right]=\delta_{r}^{\prime}=\int_{0}^{\infty} x^{r} f(x ; \beta) d x \\
E\left[X^{r}\right]=\sum_{j=0}^{\infty} \sum_{k=0}^{\infty} \sum_{l=0}^{k} \sum_{m=0}^{\infty} t_{h} \int_{0}^{\infty} x^{r} \Phi_{\theta(m+1)} d x \\
E\left[X^{r}\right]=\delta_{r}^{\prime}=\sum_{j=0}^{\infty} \sum_{k=0}^{\infty} \sum_{l=0}^{k} \sum_{m=0}^{\infty} t_{h} I_{\theta(m+1)}
\end{gathered}
$$

where $t_{h}$ is as equation (19) and

$$
I_{\theta(m+1)}=\int_{0}^{\infty} x^{r} \Phi_{\theta(m+1)} d x
$$

is the moment of the Exp-G distribution with power parameter $\theta(m+1)$.

Furthermore, the $n^{\text {th }}$ central moment of $\mathrm{X}$, given as $F_{n}$, is

$$
\begin{gathered}
F_{n}=E\left[\left(X-\delta_{r}^{\prime}\right)^{n}\right]=\sum_{r=0}^{n}\left(\begin{array}{l}
n \\
r
\end{array}\right)\left(-\delta_{1}^{\prime}\right)^{n-r} E\left[X^{r}\right] \\
=\sum_{r=0}^{n}\left(\begin{array}{l}
n \\
r
\end{array}\right)(-1)^{n-r}\left(\delta_{1}\right)^{n-r} E\left[X^{r}\right] .
\end{gathered}
$$

Therefore, the $n^{\text {th }}$ central moment of $\mathrm{X}$ is

$$
\begin{aligned}
F_{n} & =\sum_{j=0}^{\infty} \sum_{k=0}^{\infty} \sum_{l=0}^{k} \sum_{m=0}^{\infty} \sum_{r=0}^{n}(-1)^{j+l+n-r}\left(\begin{array}{c}
\gamma-1 \\
j
\end{array}\right)\left(\begin{array}{c}
k \\
l
\end{array}\right)\left(\begin{array}{l}
n \\
r
\end{array}\right) \frac{(j+1)^{k} \tau^{k}(l+1)^{m}}{k ! m !(m+1)} \gamma A^{\gamma} \tau I_{\theta(m+1)} \\
& =\sum_{j=0}^{\infty} \sum_{k=0}^{\infty} \sum_{l=0}^{k} \sum_{m=0}^{\infty} \sum_{r=0}^{n} r_{n} I_{\theta(m+1)}
\end{aligned}
$$

where

$$
r_{n}=(-1)^{j+l+n-r}\left(\begin{array}{c}
\gamma-1 \\
j
\end{array}\right)\left(\begin{array}{l}
k \\
l
\end{array}\right)\left(\begin{array}{l}
n \\
r
\end{array}\right) \frac{(j+1)^{k} \tau^{k}(l+1)^{m}}{k ! m !(m+1)} \gamma A^{\gamma} \tau
$$

and $I_{\theta(m+1)}$ is in (23).

The variance(V), skewness $(\mathrm{S})$ and kurtosis $(\mathrm{K})$ measures can be calculated from the ordinary moments using well-known relationships as in

$$
\begin{gathered}
V=\delta_{2}^{\prime}-\left(\delta_{1}^{\prime}\right)^{2} \\
S=\frac{\delta_{3}^{\prime}}{\delta_{2}^{\frac{3}{2}}}
\end{gathered}
$$


and

$$
K=\frac{\delta_{4}^{\prime}}{\delta_{2}^{2}}
$$

where $\delta_{1}^{\prime}$ is the first moment (mean), $\delta_{2}$ ' is the second moment and $\delta_{4}^{\prime}$ is the fourth moment about the mean.

\subsection{Moment generating function}

The moment generating function $M_{X}(t)$ of $\mathrm{X}$ is

$$
\begin{gathered}
M_{X}(t)=\int_{0}^{\infty} e^{t x} f(x ; \beta) d x \\
M_{X}(t)=\sum_{j=0}^{\infty} \sum_{k=0}^{\infty} \sum_{l=0}^{k} \sum_{m=0}^{\infty} t_{h} \int_{0}^{\infty} e^{t x} \theta(m+1) g(x ; \beta) G(x ; \beta)^{\theta(m+1)-1} \\
M_{X}(t)=\sum_{j=0}^{\infty} \sum_{k=0}^{\infty} \sum_{l=0}^{k} \sum_{m=0}^{\infty} t_{h} \Gamma_{\theta(m+1)}
\end{gathered}
$$

where $\Gamma_{\theta(m+1)}$ is the moment generating function of the Exp-G distribution with $\theta(\mathrm{m}+1)$ power parameter.

\subsection{Quantile function}

The quantile function $\left(x_{q}\right)$ of the Exponentiated Chen $\mathrm{G}$ family of distributions is obtained by inverting the cdf of the Exponentiated Chen $\mathrm{G}$ distribution into U, where $\mathrm{U}$ is distributed to $\operatorname{Uniform}(0,1), 0 \leq \mathrm{u} \leq 1$. This results to

$$
x_{q}=G^{-1}\left(\left(\ln \left(1-\left(\frac{1}{\tau} \ln \left(1-\frac{u^{\frac{1}{\gamma}}}{A}\right)\right)\right)\right)^{\frac{1}{\theta}} ; \beta\right)
$$

With this function, random numbers for the parameters of the model can be generated. This is used for simulation studies purposes when the function $\mathrm{G}(\mathrm{x} ; \beta)$ is specified.

\subsection{Renyi entropy}

In Information Theory, Renyi entropy generalizes Collision entropy, Shannon entropy, and Hartley entropy. Entropies quantify the uncertainty, randomness, or diversity of a system. This property is very important in ecology and statistics as indices of diversity. Renyi entropy is also important in quantum information, where it can be used as a measure of entanglement. Renyi Entropy is expressed as

$$
I_{R}(\pi)=\frac{1}{1-\pi} \log \left[\int_{0}^{\infty} f^{\pi}(x ; \beta) d x\right] .
$$

Therefore, the Renyi entropy of the ECG is defined by

$$
I_{R}(\pi)=\frac{1}{1-\pi} \log \int_{0}^{\infty} \gamma^{\pi} A^{\pi \gamma} \tau^{\pi} \theta^{\pi} g(x ; \beta)^{\pi} G(x ; \beta)^{\pi(\theta-1)} e^{\pi G(x ; \beta)^{\theta}} e^{\pi \tau\left(1-e^{G(x ; \beta)^{\theta}}\right)}\left[1-e^{\tau\left(1-e^{G(x ; \beta)^{\theta}}\right)}\right]^{\pi(\gamma-1)} .
$$

To define Renyi entropy in terms of the Exp-G density function, consider using the power series expansion

$$
\left[1-e^{\tau\left(1-e^{G(x ; \beta)^{\theta}}\right)}\right]^{\pi(\gamma-1)}=\sum_{j=0}^{\infty}(-1)^{j}\left(\begin{array}{c}
\pi(\gamma-1) \\
j
\end{array}\right) e^{j \tau\left(1-e^{G(x ; \beta)^{\theta}}\right)}
$$




$$
\begin{gathered}
I_{R}(\pi)=\frac{1}{1-\pi} \log \left[\int_{0}^{\infty} \gamma^{\pi} A^{\pi \gamma} \tau^{\pi} \theta^{\pi} g(x ; \beta)^{\pi} G(x ; \beta)^{\pi(\theta-1)} e^{\pi G(x ; \beta)^{\theta}} \sum_{j=0}^{\infty}(-1)^{j}\left(\begin{array}{c}
\pi(\gamma-1) \\
j
\end{array}\right)\right] \\
e^{(j+\pi) \tau\left(1-e^{G(x ; \beta)}\right)} \\
e^{(j+\pi) \tau\left(1-e^{G(x ; \beta)^{\theta}}\right)}=\sum_{k=0}^{\infty} \frac{(j+\pi)^{k} \tau^{k}\left(1-e^{G(x ; \beta)^{\theta}}\right)^{k}}{k !}
\end{gathered}
$$

Inserting equation (32) in (31),

$$
I_{R}(\pi)=\frac{1}{1-\pi} \log \int_{0}^{\infty} \gamma^{\pi} A^{\pi \gamma} \tau^{\pi} \theta^{\pi} g(x ; \beta)^{\pi} G(x ; \beta)^{\pi(\theta-1)} e^{\pi G(x ; \beta)^{\theta}} \sum_{j=0}^{\infty} \sum_{k=0}^{\infty}(-1)^{j} \frac{(j+\pi)^{k} \tau^{k}\left(1-e^{\left.G(x ; \beta)^{\theta}\right)^{k}}\right.}{k !} .
$$

From

$$
\left(1-e^{G(x ; \beta)^{\theta}}\right)^{k}=\sum_{l=0}^{k}(-1)^{l}\left(\begin{array}{l}
k \\
l
\end{array}\right) e^{l G(x ; \beta)^{\theta}}
$$

it further gives

$$
I_{R}(\pi)=\frac{1}{1-\pi} \log \int_{0}^{\infty} \gamma^{\pi} A^{\pi \gamma} \tau^{\pi} \theta^{\pi} g(x ; \beta)^{\pi} G(x ; \beta)^{\pi(\theta-1)}
$$

from the expression

$$
\begin{gathered}
\sum_{j=0}^{\infty} \sum_{k=0}^{\infty} \sum_{l=0}^{k}(-1)^{j+l}\left(\begin{array}{c}
\pi(\gamma-1) \\
j
\end{array}\right)\left(\begin{array}{c}
k \\
l
\end{array}\right) \frac{(j+\pi)^{k} \tau^{k}}{k !} e^{(l+\pi) G(x ; \beta)^{\theta}} \\
e^{(l+\pi) G(x ; \beta)^{\theta}}=\sum_{m=0}^{\infty} \frac{(l+\pi)^{m} G(x ; \beta)^{m \theta}}{m !} \\
I_{R}(\pi)=\frac{1}{1-\pi} \log \int_{0}^{\infty} \sum_{j=0}^{\infty} \sum_{k=0}^{\infty} \sum_{l=0}^{k} \sum_{m=0}^{\infty}(-1)^{j+l}\left(\begin{array}{c}
\pi(\gamma-1) \\
j
\end{array}\right)\left(\begin{array}{c}
k \\
l
\end{array}\right) \frac{(j+\pi)^{k} \tau^{k}(l+\pi)^{m}}{k ! m !} \\
\gamma^{\pi} A^{\pi \gamma} \tau^{\pi} \theta^{\pi} g(x ; \beta)^{\pi} G(x ; \beta)^{\theta(m+\pi)-\pi}
\end{gathered}
$$

which is also

$$
\begin{gathered}
I_{R}(\pi)=\frac{1}{1-\pi} \log \sum_{j=0}^{\infty} \sum_{k=0}^{\infty} \sum_{l=0}^{k} \sum_{m=0}^{\infty}(-1)^{j+l}\left(\begin{array}{c}
\pi(\gamma-1) \\
j
\end{array}\right)\left(\begin{array}{c}
k \\
l
\end{array}\right) \frac{(j+\pi)^{k} \tau^{k}(l+\pi)^{m}}{k ! m !} \\
\gamma^{\pi} A^{\pi \gamma} \tau^{\pi} \theta^{\pi}\left(\int_{0}^{\infty}\left(\frac{\theta(m+\pi)}{\pi}-1\right) g(x ; \beta) G(x ; \beta)^{\frac{\theta(m+\pi)}{\pi}-1}\right)^{\pi} d x \\
I_{R}(\pi)=\frac{1}{1-\pi} \log \int_{0}^{\infty} \sum_{j=0}^{\infty} \sum_{k=0}^{\infty} \sum_{l=0}^{k} \sum_{m=0}^{\infty} u_{h} \Lambda^{\pi} d x
\end{gathered}
$$

where

$$
u_{h}=(-1)^{j+l}\left(\begin{array}{c}
\pi(\gamma-1) \\
j
\end{array}\right)\left(\begin{array}{l}
k \\
l
\end{array}\right) \frac{(j+\pi)^{k} \tau^{k}(l+\pi)^{m}}{k ! m !} \gamma^{\pi} A^{\pi \gamma} \tau^{\pi} \theta^{\pi}
$$

and

$$
\Lambda=\frac{\theta(m+\pi)}{\pi} g(x ; \beta) G(x ; \beta)^{\frac{\theta(m+\pi)}{\pi}-1} d x
$$

$\Gamma$ is pdf of the Exp-G distribution with $\frac{\theta(m+\pi)}{\pi}$ power parameter. Thus, the Renyi entropy of the ECG family of distributions is established in terms of the entropy of the Exp-G distribution. 


\subsection{Survival function and hazard function}

The survival function $\mathrm{s}(\mathrm{x} ; \beta)$ and the hazard function $\mathrm{h}(\mathrm{x} ; \beta)$ of the Exponentiated Chen-G family of distributions is derived as

$$
s(x ; \beta)=1-\left(A\left[1-e^{\tau\left(1-e^{G(x ; \beta)^{\theta}}\right)}\right]\right)^{\gamma}
$$

and

$$
h(x ; \beta)=\frac{\gamma A^{\gamma} \tau \theta g(x ; \beta) G(x ; \beta)^{\theta-1} e^{G(x ; \beta)^{\theta}} e^{\tau\left(1-e^{G(x ; \beta)^{\theta}}\right)}\left[1-e^{\tau\left(1-e^{G(x ; \beta)^{\theta}}\right)}\right]^{\gamma-1}}{1-\left(A\left[1-e^{\tau\left(1-e^{G(x ; \beta)^{\theta}}\right)}\right]\right)^{\gamma}}
$$

respectively. The reverse hazard function $\bar{h}(x ; \beta)$ is obtained as

$$
\bar{h}(x ; \beta)=\frac{\gamma A^{\gamma-1} \tau \theta g(x ; \beta) G(x ; \beta)^{\theta-1} e^{G(x ; \beta)^{\theta}} e^{\tau\left(1-e^{G(x ; \beta)^{\theta}}\right)}}{\left[1-e^{\tau\left(1-e^{G(x ; \beta)^{\theta}}\right)}\right]} .
$$

\subsection{Order statistics}

Let $X_{1}, \ldots, X_{n}$ be a random sample from the Exponentiated Chen-G family of distributions, the pdf of $i^{\text {th }}$ order statistic, say $X_{i: n}$, can be written as

$$
f_{i: n}\left(x_{i} ; \beta\right)=\frac{1}{B(i, n-i+1)} f\left(x_{i} ; \beta\right) \sum_{v=0}^{n-1}(-1)^{v}\left(\begin{array}{c}
n-1 \\
v
\end{array}\right) F\left(x_{i} ; \beta\right)^{v+i-1} .
$$

Therefore

$$
F\left(x_{i} ; \beta\right)^{v+i-1}=\left(\sum_{j=0}^{\infty} \sum_{k=0}^{\infty} \sum_{l=0}^{k} \sum_{m=0}^{\infty} t_{h} \lambda_{\theta(m+1)}\right)^{v+i-1}
$$

which is

$$
F\left(x_{i} ; \beta\right)^{v+i-1}=\left(\sum_{j=0}^{\infty} \sum_{k=0}^{\infty} \sum_{l=0}^{k} \sum_{m=0}^{\infty} t_{h}\left[R^{m+1}\right]^{\theta}\right)^{v+i-1} .
$$

Hence, using an equation as explored in the work of Gradshteyn and Ryzhik (2007) for a power series that is raised to a power of a positive integer $n$ :

$$
\left(\sum_{k=0}^{\infty} f_{k} u^{k}\right)^{n}=\sum_{k=0}^{\infty} C_{n, k} u^{k}
$$

where coefficient $C_{n, k}$ can be derived from the recurrence equation

$$
C_{n, k}=\left(k\left(C_{n, 0}\right)^{\frac{1}{n}}\right)^{-1} \sum_{l=1}^{k}(l(n+1)-r)\left(\left(C_{n, l}\right)^{\frac{1}{n}}\right)\left(C_{n, k-m}\right) .
$$

Therefore using (38) and (39),

$$
F\left(x_{i} ; \beta\right)^{j+i-1}=\sum_{j=0}^{\infty} \sum_{k=0}^{\infty} \sum_{l=0}^{k} \sum_{m=0}^{\infty} C_{h, i+j-1}\left[R^{m+1}\right]^{\theta} .
$$

Substituting (18) and (39) into (36), and using the power series expansion, the pdf of $X_{i: n}$ of the ECG is derived as

$$
f_{i: n}\left(x_{i}\right)=\frac{1}{B(i, n-i+1)} \sum_{v=0}^{n-1}(-1)^{v}\left(\begin{array}{c}
n-i \\
v
\end{array}\right) \sum_{j=0}^{\infty} \sum_{k=0}^{\infty} \sum_{l=0}^{k} \sum_{m=0}^{\infty} C_{h, i+j-1} Z^{\theta(m+1)}
$$


where $Z^{\theta(m+1)}$ is the Exp-G pdf with power $\theta(m+1)$.

Therefore, the first order $X_{1}$ of the pdf of the ECG distribution is

$$
f_{1: n}(x)=n \sum_{v=0}^{n-1}(-1)^{v}\left(\begin{array}{c}
n-1 \\
v
\end{array}\right) \sum_{j=0}^{\infty} \sum_{k=0}^{\infty} \sum_{l=0}^{k} \sum_{m=0}^{\infty} C_{h, j} Z^{\theta(m+1)}
$$

and the last order $X_{n}$ as

$$
f_{n: n}(x)=n \sum_{v=0}^{n-1}(-1)^{v}\left(\begin{array}{c}
n-i \\
v
\end{array}\right) \sum_{j=0}^{\infty} \sum_{k=0}^{\infty} \sum_{l=0}^{k} \sum_{m=0}^{\infty} C_{h, n+j-1} Z^{\theta(m+1)} .
$$

\section{Maximum likelihood estimation}

In this section, the estimation of the ECG family under complete observation and censored observation is studied. This is done using the maximum likelihood estimation method. Let $X_{i}, \ldots, X_{n}$ be a random sample from the Exponentiated Chen-G family of distributions with observed values $x_{i}, \ldots, x_{n}$ and $\zeta=(\tau, \theta, \gamma, \beta)$ be parameter vector. Then, the log-Likelihood function of Exponentiated Chen- $G$ family of distributions under complete observation is obtained as

$$
\begin{aligned}
l\left(x_{i} ; \tau, \theta, \gamma, \beta\right) & =l=n \ln \gamma+n \gamma \ln A+n \ln \tau+n \ln \theta+\sum_{i=1}^{n} \ln g\left(x_{i} ; \beta\right)+(\theta-1) \sum_{i=1}^{n} \ln G\left(x_{i} ; \beta\right) \\
& +\sum_{i=1}^{n} \ln G\left(x_{i} ; \beta\right)^{\theta}+\sum_{i=1}^{n} \tau\left(1-e^{G\left(x_{i} ; \beta\right)^{\theta}}\right)+(\gamma-1) \sum_{i=1}^{n} \ln \left(1-e^{\tau\left(1-e^{G\left(x_{i} ; \beta\right)^{\theta}}\right)}\right) .
\end{aligned}
$$

Differentiating the loglikelihood function with respect to $\zeta$ gives

$$
\begin{gathered}
\frac{\partial l}{\partial \gamma}=\frac{n}{\gamma}+\ln A+\ln \left(1-e^{\tau\left(1-e^{G\left(x_{i} ; \beta\right)^{\theta}}\right)}\right) \\
\frac{\partial l}{\partial \tau}=\frac{n}{\tau}+n \gamma \frac{A^{\prime}}{A}+\sum_{i=1}^{n}\left(1-e^{G\left(x_{i} ; \beta\right)^{\theta}}\right)+(\gamma-1) \sum_{i=1}^{n} \frac{\left.\left(e^{G\left(x_{i} ; \beta\right)^{\theta}}-1\right) e^{\tau\left(1-e^{G\left(x_{i} ; \beta\right)^{\theta}}\right.}\right)}{1-e^{\tau\left(1-e^{G\left(x_{i} ; \beta\right)^{\theta}}\right)}} \\
\frac{\partial l}{\partial \theta}=\frac{n}{\theta}+\sum_{i=1}^{n} \ln G\left(x_{i} ; \beta\right)+\sum_{i=1}^{n} \frac{G\left(x_{i} ; \beta\right)^{\theta} \ln G\left(x_{i} ; \beta\right)}{G\left(x_{i} ; \beta\right)^{\theta}} \\
+(\gamma-1) \sum_{i=1}^{n} \frac{\tau G\left(x_{i} ; \beta\right)^{\theta} \ln G\left(x_{i} ; \beta\right) e^{G\left(x_{i} ; \beta\right)^{\theta}} e^{\tau\left(1-e^{G\left(x_{i} ; \beta\right)^{\theta}}\right)}}{1-e^{\tau\left(1-e^{G\left(x_{i} ; \beta\right)^{\theta}}\right)}} \\
\frac{\partial l}{\partial \beta}=\sum_{i=1}^{n} \frac{g^{\prime}\left(x_{i} ; \beta\right)}{g\left(x_{i} ; \beta\right)}+(\theta-1) \sum_{i=1}^{n} \frac{G^{\prime}\left(x_{i} ; \beta\right)}{G\left(x_{i} ; \beta\right)}+\sum_{i=1}^{n} \frac{\theta G\left(x_{i} ; \beta\right)^{\theta-1} G^{\prime}\left(x_{i} ; \beta\right)}{G\left(x_{i} ; \beta\right)^{\theta}} \\
+\sum_{i=1}^{n} \tau G^{\prime}\left(x_{i} ; \beta\right) G\left(x_{i} ; \beta\right)^{\theta-1} e^{G\left(x_{i} ; \beta\right)^{\theta}}+(\gamma-1) \sum_{i=1}^{n} \frac{G^{\prime}\left(x_{i} ; \beta\right) G\left(x_{i} ; \beta\right)^{\theta-1} e^{G\left(x_{i} ; \beta\right)^{\theta}}}{1-e^{\tau\left(1-e^{G\left(x_{i} ; \beta\right)^{\theta}}\right)}}
\end{gathered}
$$

where $G^{\prime}\left(x_{i} ; \beta\right)=\frac{\partial G\left(x_{i} ; \beta\right)}{\partial \beta}, A=\frac{1}{1-e^{\tau(1-e)}}$ and $\mathrm{A}^{\prime}=\frac{e^{\tau(1-e)}(1-e)}{\left(1-e^{\tau(1-e)}\right)^{2}}$.

The maximum likelihood estimators of the population parameters are obtained by setting the non-linear equations in equations (42)-(45) to zero. These equations are very difficult to 
obtain, so iterative procedures are used. Furthermore, in the case of interval estimation, the following observed information matrix is required

$$
U(f, s)=\left(\begin{array}{cccc}
U_{\gamma \gamma} & U_{\gamma \tau} & U_{\gamma \theta} \ldots . & U_{\gamma \beta} \\
U_{\tau \gamma} & U_{\tau \tau} & U_{\tau \theta} \ldots & U_{\tau \beta} \\
U_{\theta \gamma} & U_{\theta \tau} & U_{\theta \theta} \ldots . & U_{\theta \beta} \\
\cdot & \cdot & \cdot & \cdot \\
\cdot & \cdot & \cdot & \cdot \\
U_{\beta \gamma} & U_{\beta \tau} & U_{\beta \theta} \ldots . & U_{\beta \beta}
\end{array}\right)
$$

where $U_{r s}=\frac{\partial^{2} l}{\partial f \partial s}$ and $\mathrm{f}, \mathrm{s}=(\gamma, \tau, \theta, \beta) U_{f, s}$ can be computed numerically. Under standard regularity conditions (Cox and Hinkley 1979), the distribution of $(\hat{\zeta}-\zeta)$ by the multivariate normal $N_{3+r}\left(0, \mathrm{~J}\left(\zeta^{-1}\right)\right.$ distribution can be estimated, where $\mathrm{r}$ is the number of parameters of the baseline distribution. The maximum values of the unrestricted and restricted loglikelihoods can be computed, to construct likelihood ratio (LR) statistics for testing some sub-models of the ECG family of distributions.

For right-censored situations, to estimate the parameters of the model, the likelihood function is given as

$$
L=\prod_{i=1}^{r} f\left(x_{i}, \beta\right) \prod_{i=r+1}^{n} s\left(x_{i}^{+}, \beta\right)
$$

where $\prod_{i=1}^{r} f\left(x_{i} ; \beta\right)$ represents the joint probability of observing the uncensored survival times, and $\prod_{i=r+1}^{n} s\left(x^{+} ; \beta\right)$ represents the joint probability of those censored survival times. Therefore, $r$ is the number of uncensored subjects and $n-r$ is for the censored subject. The censored likelihood $\mathrm{L}(\zeta)^{*}$ for the model parameters is

$$
\begin{aligned}
l\left(x_{i}, x_{i}^{+} ; \zeta^{*}\right) & =l^{*}=r \ln \gamma+n \gamma \ln A+r \ln \tau+r \ln \theta+\sum_{i=1}^{r} \ln g\left(x_{i} ; \beta\right)+(\theta-1) \sum_{i=1}^{r} \ln G\left(x_{i} ; \beta\right) \\
& +\sum_{i=1}^{r} \ln G\left(x_{i} ; \beta\right)^{\theta}+\sum_{i=1}^{r} \tau\left(1-e^{G\left(x_{i} ; \beta\right)^{\theta}}\right)+(\gamma-1) \sum_{i=1}^{r} \ln \left(1-e^{\tau\left(1-e^{G\left(x_{i}^{+} ; \beta\right)^{\theta}}\right)}\right) \\
& +\sum_{i=r+1}^{n} \ln \left(1-\left(A\left[1-e^{\tau\left(1-e^{G\left(x_{i}^{+} ; \beta\right)^{\theta}}\right)}\right]\right)^{\gamma}\right),
\end{aligned}
$$

$$
\begin{aligned}
& \frac{\partial l^{*}}{\partial \gamma}=\frac{r}{\gamma}+\ln A+\sum_{i=1}^{r} \ln \left(1-e^{\tau\left(1-e^{G\left(x_{i} ; \beta\right)^{\theta}}\right)}\right)-\sum_{i=r+1}^{n} \frac{\left(A\left[1-e^{\tau\left(1-e^{G\left(x_{i}^{+} ; \beta\right)^{\theta}}\right)}\right]\right)^{\gamma} \ln A\left[1-e^{\tau\left(1-e^{G\left(x_{i}^{+} ; \beta\right)^{\theta}}\right)}\right]}{1-\left(A\left[1-e^{\tau\left(1-e^{G\left(x_{i}^{+} ; \beta\right)^{\theta}}\right)}\right]\right)^{\gamma}} \\
& \frac{\partial l}{\partial \tau}=\frac{r}{\tau}+n \gamma \frac{A^{\prime}}{A}+\sum_{i=1}^{r}\left(1-e^{G\left(x_{i} ; \beta\right)^{\theta}}\right) \\
& +(\gamma-1) \sum_{i=1}^{r} \frac{\left(e^{G\left(x_{i} ; \beta\right)^{\theta}}-1\right) e^{\tau\left(1-e^{G\left(x_{i} ; \beta\right)^{\theta}}\right)}}{1-e^{\tau\left(1-e^{G\left(x_{i} ; \beta\right)^{\theta}}\right)}} \\
& -\sum_{i=r+1}^{n} \frac{\gamma\left(A^{\prime}\left(1-e^{G\left(x_{i}^{+} ; \beta\right)^{\theta}}\right)+A\left(\left(e^{G\left(x_{i}^{+} ; \beta\right)^{\theta}}-1\right) e^{\tau\left(1-e^{G\left(x_{i}^{+} ; \beta\right)^{\theta}}\right)}\right)\right)^{\gamma-1}}{1-\left(A\left[1-e^{\tau\left(1-e^{G\left(x_{i}^{+} ; \beta\right)^{\theta}}\right)}\right]\right)^{\gamma}},
\end{aligned}
$$




$$
\begin{aligned}
& \frac{\partial l}{\partial \theta}=\frac{r}{\theta}+\sum_{i=1}^{r} \ln G\left(x_{i} ; \beta\right)+\sum_{i=1}^{r} \frac{G\left(x_{i} ; \beta\right)^{\theta} \ln G\left(x_{i} ; \beta\right)}{G\left(x_{i} ; \beta\right)^{\theta}} \\
& +(\gamma-1) \sum_{i=1}^{r} \frac{\tau G\left(x_{i} ; \beta\right)^{\theta} \ln G\left(x_{i} ; \beta\right) e^{G\left(x_{i} ; \beta\right)^{\theta}} e^{\tau\left(1-e^{G\left(x_{i} ; \beta\right)^{\theta}}\right)}}{1-e^{\tau\left(1-e^{G\left(x_{i} ; \beta\right)^{\theta}}\right)}} \\
& -\sum_{i=r+1}^{n} \frac{\gamma A^{\gamma} \tau G\left(x_{i}^{+} ; \beta\right)^{\theta} \ln G\left(x_{i}^{+} ; \beta\right) e^{\tau\left(1-e^{G\left(x_{i}^{+} ; \beta\right)^{\theta}}\right)}\left(1-e^{\tau\left(1-e^{G\left(x_{i}^{+} ; \beta\right)^{\theta}}\right)}\right)^{\gamma-1}}{1-\left(A\left[1-e^{\tau\left(1-e^{G\left(x_{i}^{+} ; \beta\right)^{\theta}}\right)}\right]\right)^{\gamma}}, \\
& \frac{\partial l^{*}}{\partial \beta}=\sum_{i=1}^{r} \frac{g^{\prime}\left(x_{i} ; \beta\right)}{g\left(x_{i} ; \beta\right)}+(\theta-1) \sum_{i=1}^{r} \frac{G^{\prime}\left(x_{i} ; \beta\right)}{G\left(x_{i} ; \beta\right)}+\sum_{i=1}^{r} \theta G\left(x_{i} ; \beta\right)^{\theta-1} G^{\prime}\left(x_{i} ; \beta\right) \\
& +\sum_{i=1}^{r} \tau G^{\prime}\left(x_{i} ; \beta\right) G\left(x_{i} ; \beta\right)^{\theta-1} e^{G\left(x_{i} ; \beta\right)^{\theta}}+(\gamma-1) \sum_{i=1}^{r} \frac{G^{\prime}\left(x_{i} ; \beta\right) G\left(x_{i} ; \beta\right)^{\theta-1} e^{G\left(x_{i} ; \beta\right)^{\theta}}}{1-e^{\tau\left(1-e^{G\left(x_{i} ; \beta\right)^{\theta}}\right)}} \\
& +\sum_{i=r+1}^{n} \frac{\gamma \tau\left(A\left(1-e^{\tau\left(1-e^{G\left(x_{i}^{+} ; \beta\right)^{\theta}}\right)}\right)\right)^{\gamma-1} e^{\tau\left(1-e^{G\left(x_{i}^{+} ; \beta\right)^{\theta}}\right)} e^{G\left(x_{i}^{+} ; \beta\right)^{\theta}} \theta G\left(x_{i} ; \beta\right)^{(\theta-1)} G^{\prime}\left(x_{i} ; \beta\right)}{1-\left(A\left[1-e^{\tau\left(1-e^{G\left(x_{i}^{+} ; \beta\right)^{\theta}}\right)}\right]\right)^{\gamma}}
\end{aligned}
$$

where $G^{\prime}\left(x_{i} ; \beta\right)=\frac{\partial G\left(x_{i} ; \beta\right)}{\partial \beta}, A=\frac{1}{1-e^{\tau(1-e)}}$ and $\mathrm{A}^{\prime}=\frac{e^{\tau(1-e)}(1-e)}{\left(1-e^{\tau(1-e)}\right)^{2}}$.

Solving equations (48),(49),(50), and (51) equal zero gives the maximum likelihood estimates of the ECG family of distributions under censored observations. This estimation can be done iteratively with the aid of statistical software.

\section{Exponentiated Chen exponential distribution}

In this section, the Exponentiated Chen Exponential distribution is introduced. This distribution is obtained by mixing the Exponentiated Chen G family of distributions and the Exponential distribution. The statistical properties, estimations, and application of the Exponentiated Chen Exponential distribution were considered. Given X as a random variable which denotes the lifetime of a system, defined by $X_{i}, X_{2}, \ldots, X_{n}$ and the distribution of each is assumed to be identically and independently distributed to the Exponential distribution, then, $\mathrm{X}$ is said to have the pdf

$$
f(x ; \mu)=\mu e^{-\mu x} \quad x>0, \mu>0
$$

and cdf

$$
F(x ; \mu)=1-e^{-\mu x} \quad x>0, \mu>0
$$

Therefore, inserting the (52) and (53) as the baseline pdf and cdf respectively in equation (1), the pdf of the Exponentiated Chen Exponential distribution is obtained as

$$
f_{E C E}(x ; \tau, \mu, \theta, \gamma)=\gamma A^{\gamma} \tau \theta\left(\mu e^{-\mu x}\right)\left(1-e^{-\mu x}\right)^{\theta-1} e^{\left(1-e^{-\mu x}\right)^{\theta}} e^{\tau\left(1-e^{\left(1-e^{-\mu x}\right)^{\theta}}\right)}\left[1-e^{\tau\left(1-e^{\left(1-e^{-\mu x}\right)^{\theta}}\right)}\right]^{\gamma-1}
$$

with the corresponding cdf as

$$
F_{E C E}(x ; \tau, \mu, \theta, \gamma)=\left(A\left[1-e^{\tau\left(1-e^{\left(1-e^{-\mu x}\right)^{\theta}}\right)}\right]\right)^{\gamma} \quad x>0, \mu, \theta, \gamma>0,|\tau|>0
$$

where $\mathrm{x}>0, \mu, \theta, \gamma>0,|\tau|>0$.

Table 1 shows the pdf and cdf of the submodels of the ECE distribution 
Table 1: Table displaying pdf and cdf of submodels of ECE distributions

\begin{tabular}{|c|c|c|c|c|}
\hline Distribution & $\mathrm{pdf}$ & & $\mathrm{cdf}$ & Author(Year) \\
\hline \multicolumn{5}{|l|}{ Exponentiated } \\
\hline Exponential(EE) & $\alpha \mu\left(1-e^{-\mu x}\right)^{\alpha-1} e^{-\mu x}$ & & $\left(1-e^{-\mu x}\right)^{\alpha}$ & Gupta et al. (1998) \\
\hline Chen Exponential(CE) & $\begin{array}{l}A \tau \theta\left(\mu e^{-\mu x}\right)(1 \\
\left.e^{-\mu x}\right)^{\theta-1} e^{\left(1-e^{-\mu x}\right)^{\theta}} \\
e^{\tau\left(1-e^{\left(1-e^{-\mu x}\right)^{\theta}}\right)}\end{array}$ & - & $A\left[1-e^{\tau\left(1-e^{\left(1-e^{-\mu x}\right)^{\theta}}\right)}\right]$ & New \\
\hline Exponential(E) & $\mu e^{-\mu x}$ & & $1-e^{-\mu x}$ & - \\
\hline
\end{tabular}

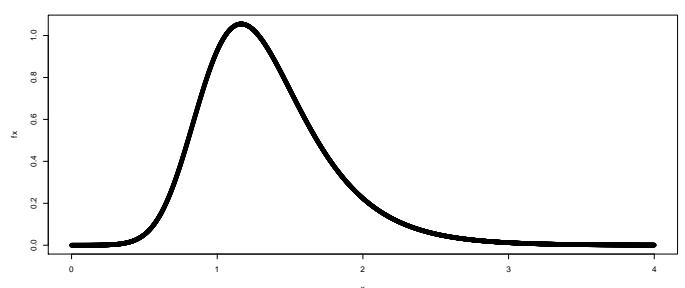

(a) $\gamma=2, \tau=-3, \theta=2, \mu=3$

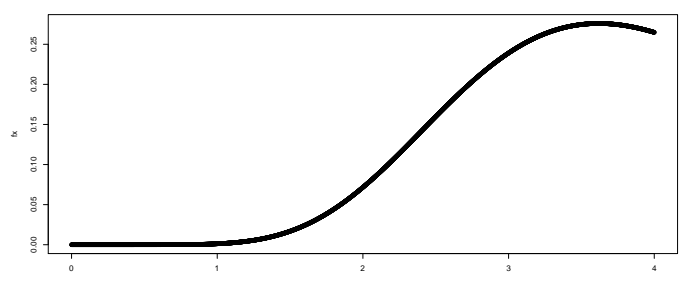

(c) $\gamma=5.2, \tau=1.3, \theta=0.2, \mu=0.056$

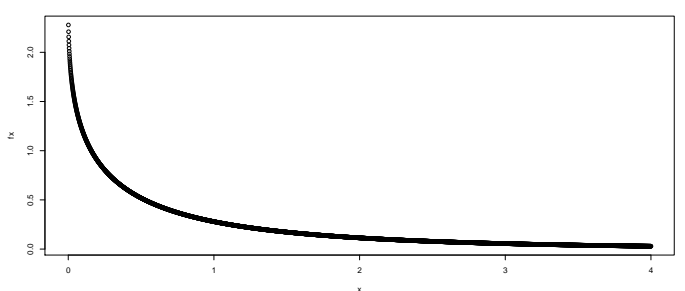

(b) $\gamma=2.2, \tau=1.3, \theta=5.2, \mu=0.56$

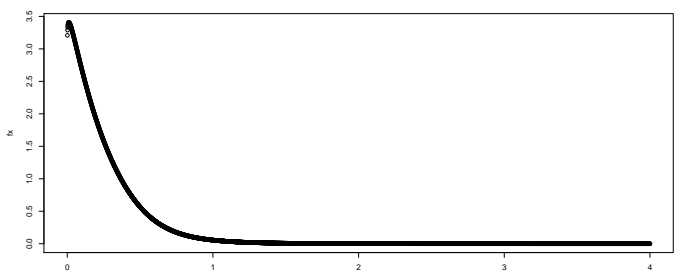

(d) $\gamma=0.2, \tau=1.3, \theta=5.2, \mu=3.6$

Figure 1: The pdf of Exponentiated Chen G Family of distributions with various parameter values

Figure 1 reveals the density plots of the ECE distribution with varying parameter values. The density plots reveal different shapes with different degrees of kurtosis and right skewness.

\subsection{Linear representation of the exponential Chen exponential distribution}

In this section, the linear presentation of the ECE distribution is obtained. Inserting the pdf and cdf of the Exponential distribution in expression 17, the mixture representation of the ECE distribution is given as

$f(x ; \beta)=\sum_{j=0}^{\infty} \sum_{k=0}^{\infty} \sum_{l=0}^{k} \sum_{m=0}^{\infty}(-1)^{j+l}\left(\begin{array}{c}\gamma-1 \\ j\end{array}\right)\left(\begin{array}{l}k \\ l\end{array}\right) \frac{(j+1)^{k} \tau^{k}(l+1)^{m}}{k ! m !(m+1)} \gamma A^{\gamma} \tau \theta(m+1)\left(\mu e^{-\mu x}\right)\left(1-e^{-\mu x}\right)^{\theta(m+1)-1}$

which is a mixture representation in form of the Exponentiated Exponential distribution. Further analyzing, using the binomial expression,

$$
\begin{gathered}
f(x ; \beta)=\sum_{j=0}^{\infty} \sum_{k=0}^{\infty} \sum_{l=0}^{k} \sum_{m=0}^{\infty} \sum_{n=0}^{\theta(m+1)-1}(-1)^{j+l+n}\left(\begin{array}{c}
\gamma-1 \\
j
\end{array}\right)\left(\begin{array}{c}
k \\
l
\end{array}\right)\left(\begin{array}{c}
\theta(m+1)-1 \\
n
\end{array}\right) \\
\frac{(j+1)^{k} \tau^{k}(l+1)^{m}}{k ! m !(m+1)} \gamma A^{\gamma} \tau \theta(m+1) \mu e^{-\mu x(n+1)}
\end{gathered}
$$




$$
\begin{gathered}
f(x ; \beta)=\sum_{j=0}^{\infty} \sum_{k=0}^{\infty} \sum_{l=0}^{k} \sum_{m=0}^{\infty} \sum_{n=0}^{\theta(m+1)-1} \sum_{p=0}^{\theta(m+1)}(-1)^{j+l+n}\left(\begin{array}{c}
\gamma-1 \\
j
\end{array}\right)\left(\begin{array}{c}
k \\
l
\end{array}\right) \\
\left(\begin{array}{c}
\theta(m+1)-1 \\
n
\end{array}\right) \frac{(j+1)^{k} \tau^{k}(l+1)^{m}}{p ! k ! m !(m+1)} \\
\gamma A^{\gamma} \tau \theta(m+1) \mu(-1)^{p} \mu^{p} x^{p}(\theta(m+1))^{p}
\end{gathered}
$$

This finally gives the linear representation of the ECE distribution as

$$
\begin{gathered}
f(x ; \beta)=\sum_{j=0}^{\infty} \sum_{k=0}^{\infty} \sum_{l=0}^{k} \sum_{m=0}^{\infty} \sum_{n=0}^{\theta(m+1)} \sum_{p=0}^{\theta(m+1)}(-1)^{j+l+n+p}\left(\begin{array}{c}
\gamma-1 \\
j
\end{array}\right)\left(\begin{array}{l}
k \\
l
\end{array}\right) \\
\left(\begin{array}{c}
\theta(m+1)-1 \\
n
\end{array}\right) \frac{(j+1)^{k} \tau^{k}(l+1)^{m}}{k ! m !(m+1) p !} \gamma A^{\gamma} \tau(\theta(m+1))^{p+1} \mu^{p+1} x^{p}
\end{gathered}
$$

Which can re-written as

$$
f(x ; \beta)=\sum_{j=0}^{\infty} \sum_{k=0}^{\infty} \sum_{l=0}^{k} \sum_{m=0}^{\infty} \sum_{n=0}^{\theta(m+1)} \sum_{p=0}^{-1} e_{h} x^{p}
$$

where

$$
\begin{gathered}
e_{h}=(-1)^{j+l+n+p}\left(\begin{array}{c}
\gamma-1 \\
j
\end{array}\right)\left(\begin{array}{l}
k \\
l
\end{array}\right)\left(\begin{array}{c}
\theta(m+1)-1 \\
n
\end{array}\right) \frac{(j+1)^{k} \tau^{k}(l+1)^{m}}{k ! m !(m+1) p !} \\
\gamma A^{\gamma} \tau(\theta(m+1))^{p+1} \mu^{p+1} .
\end{gathered}
$$

\subsection{Quantile function of ECE distribution}

For the quantile function of the Exponentiated Chen Exponential distribution, the cdf of the Exponential distribution is inverted in expression (4.3). Therefore the quantile function is obtained as

$$
1-e^{-\mu x_{q}}=\left(\left(\ln \left(1-\left(\frac{1}{\tau} \ln \left(1-\frac{u^{\frac{1}{\gamma}}}{A}\right)\right)\right)\right)^{\frac{1}{\theta}}\right)
$$

which gives

$$
x_{q}=-\frac{\ln \left(1-\left(\left(\ln \left(1-\left(\frac{1}{\tau} \ln \left(1-\frac{u^{\frac{1}{\gamma}}}{A}\right)\right)\right)\right)^{\frac{1}{\theta}}\right)\right.}{\mu} .
$$

Therefore, random numbers for the parameters of the ECE model can be generated using the expression in (62).

\subsection{Survival and hazard functions}

The ECE distribution has the survival function, $S_{E C E}$, as

$$
S_{E C E}=1-\left\{\left(A\left[1-e^{\tau\left(1-e^{\left(1-e^{-\mu x}\right)^{\theta}}\right)}\right]\right)^{\gamma}\right\}
$$

and the hazard $h_{E C E}$ function as

$$
h_{E C E}=\frac{\gamma A^{\gamma} \tau \theta\left(\mu e^{-\mu x}\right)\left(1-e^{-\mu x}\right)^{\theta-1} e^{\left(1-e^{-\mu x}\right)^{\theta}} e^{\tau\left(1-e^{\left(1-e^{-\mu x}\right)^{\theta}}\right.}\left[1-e^{\tau\left(1-e^{\left(1-e^{-\mu x}\right)^{\theta}}\right)}\right]^{\gamma-1}}{\left(A\left[1-e^{\tau\left(1-e^{\left(1-e^{-\mu x}\right)^{\theta}}\right)}\right]\right)^{\gamma}} .
$$




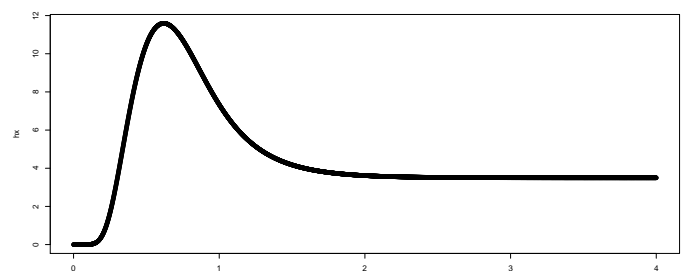

(a) $\gamma=2, \tau=5.5, \theta=5.5, \mu=3.56$

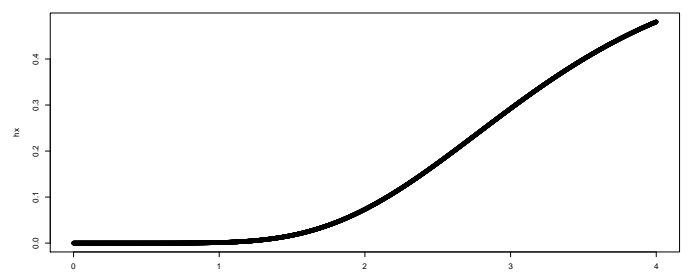

(c) $\gamma=6.2, \tau=2.3, \theta=1.2, \mu=0.56$

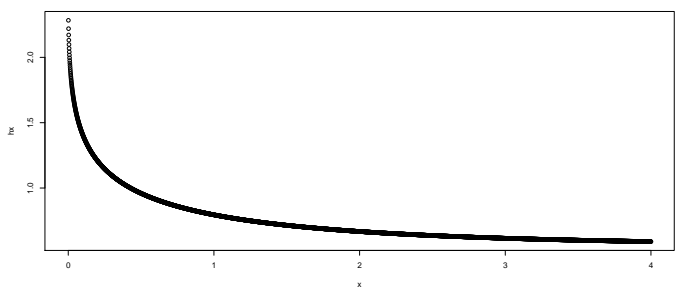

(b) $\gamma=5.2, \tau=1.3, \theta=0.2, \mu=1.56$

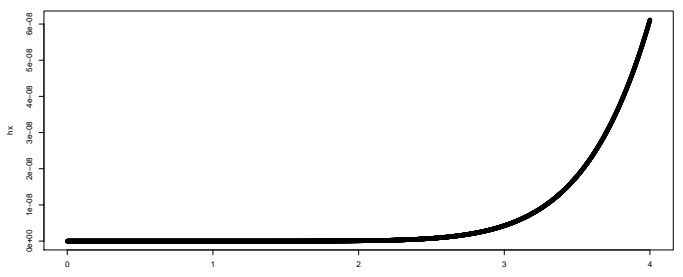

(d) $\gamma=0.2, \tau=1.3, \theta=5.2, \mu=3.6$

Figure 2: Hazard function of Exponentiated Chen Exponential Distribution with various parameter values

Figure 2 reveals the hazard function plots of the ECE distribution with varying parameter values. The hazard rate function for some selected values exhibited upside-down bathtub, decreasing and increasing failure rates.

\subsection{Moments and moment generating function}

From the expression in (26), the $r^{t h}$ moment of the ECE distribution is obtained by

$$
E\left[X^{r}\right]=\delta_{r}^{\prime}=\sum_{j=0}^{\infty} \sum_{k=0}^{\infty} \sum_{l=0}^{k} \sum_{m=0}^{\infty} t_{h} I_{\theta(m+1)}
$$

where $t_{h}$ is as equation (19) and $I_{\theta(m+1)}$ is in (23).$/ /$ Therefore,

$$
I_{\theta(m+1)}=\int_{0}^{\infty} x^{r} \theta(m+1) \mu e^{-\mu x}\left(1-e^{-\mu x}\right)^{\theta(m+1)-1} d x
$$

From

$$
\left(1-e^{-\mu x}\right)^{\theta(m+1)-1}=\sum_{p=0}^{\infty}(-1)^{p}\left(\begin{array}{c}
\theta(m+1)-1 \\
p
\end{array}\right) e^{-\mu p x}
$$

Inserting (65) in (64) to have

$$
\begin{gathered}
I_{\theta(m+1)}=\sum_{p=0}^{\infty}(-1)^{p}\left(\begin{array}{c}
\theta(m+1)-1 \\
p
\end{array}\right) \mu \theta(m+1) \int_{0}^{\infty} x^{r} e^{-\mu x(p+1)} d x \\
\int_{0}^{\infty} x^{r} e^{-\mu x(p+1)} d x=\frac{\Gamma(r+1)}{(\mu(p+1))^{r+1}} \\
I_{\theta(m+1)}=\sum_{p=0}^{\infty}(-1)^{p}\left(\begin{array}{c}
\theta(m+1)-1 \\
p
\end{array}\right) \mu \theta(m+1) \frac{\Gamma(r+1)}{(\mu(p+1))^{r+1}}
\end{gathered}
$$

Therefore

$$
E\left[X^{r}\right]=\sum_{j=0}^{\infty} \sum_{k=0}^{\infty} \sum_{l=0}^{k} \sum_{m=0}^{\infty} \sum_{n=0}^{\theta(m+1)} \sum_{p=0}^{-1} t_{h}(-1)^{p}\left(\begin{array}{c}
\theta(m+1)-1 \\
p
\end{array}\right) \mu \theta(m+1) \frac{\Gamma(r+1)}{(\mu(p+1))^{r+1}}
$$


Furthermore, the $n^{\text {th }}$ central moment of X given that it follows the ECE distribution is

$$
\begin{gathered}
F_{n}=E\left[\left(X-\delta_{r}^{\prime}\right)^{n}\right]=\sum_{r=0}^{n}\left(\begin{array}{l}
n \\
r
\end{array}\right)\left(-\delta_{1}^{\prime}\right)^{n-r} \sum_{j=0}^{\infty} \sum_{k=0}^{\infty} \sum_{l=0}^{k} \sum_{m=0}^{\infty} \sum_{n=0}^{\theta(m+1)} \sum_{p=0}^{\theta(m+1)} t_{h}(-1)^{p}\left(\begin{array}{c}
\theta(m+1)-1 \\
p
\end{array}\right) \\
\mu \theta(m+1) \frac{\Gamma(r+1)}{(\mu(p+1))^{r+1}}
\end{gathered}
$$

where

$$
\delta_{1}=\sum_{j=0}^{\infty} \sum_{k=0}^{\infty} \sum_{l=0}^{k} \sum_{m=0}^{\infty} \sum_{n=0}^{\theta(m+1)} \sum_{p=0}^{-1} t_{h}(-1)^{p}\left(\begin{array}{c}
\theta(m+1)-1 \\
p
\end{array}\right) \mu \theta(m+1) \frac{1}{(\mu(p+1))^{2}}
$$

For the moment generating function, using the expression in (28), the moment generating function $M_{X}(t)$ of ECE distribution is

$$
M_{X}(t)=\sum_{j=0}^{\infty} \sum_{k=0}^{\infty} \sum_{l=0}^{k} \sum_{m=0}^{\infty} t_{h} \int_{0}^{\infty} e^{t x} \theta(m+1)\left(\mu e^{-\mu x}\right)\left(1-e^{-\mu x}\right)^{\theta(m+1)-1} d x
$$

From (65)

$$
\begin{gathered}
\int_{0}^{\infty} e^{t x} \theta(m+1)\left(\mu e^{-\mu x}\right)\left(1-e^{-\mu x}\right)^{\theta(m+1)-1}=\sum_{p=0}^{\infty}(-1)^{p}\left(\begin{array}{c}
\theta(m+1)-1 \\
p
\end{array}\right) \mu \theta(m+1) \int_{0}^{\infty} e^{t x} e^{-x(\mu(p+1))} d x \\
=\sum_{p=0}^{\infty}(-1)^{p}\left(\begin{array}{c}
\theta(m+1)-1 \\
p
\end{array}\right) \mu \theta(m+1) \int_{0}^{\infty} e^{-x(\mu(p+1)-t)} d x \\
=\sum_{p=0}^{\infty}(-1)^{p}\left(\begin{array}{c}
\theta(m+1)-1 \\
p
\end{array}\right) \mu \theta(m+1) \frac{1}{\mu(p+1)-t} .
\end{gathered}
$$

Therefore, the m.g.f of the ECE distribution is

$$
M_{X}(t)=\sum_{j=0}^{\infty} \sum_{k=0}^{\infty} \sum_{l=0}^{k} \sum_{m=0}^{\infty} \sum_{p=0}^{\infty} t_{h}(-1)^{p}\left(\begin{array}{c}
\theta(m+1)-1 \\
p
\end{array}\right) \mu \theta(m+1) \frac{1}{\mu(p+1)-t} .
$$

Tables 2, 3, 4, and 5 show different mean, variance, skewness, and kurtosis of values of the ECE distribution. From table 3, all values are positive, in table 4, the values are positive, indicating the ability of the distribution to handle real-life situations which are most times rightly skewed. Positive and negative values in table 5 show that it can handle both heavy tail and light tail date. 
Table 2: Mean of ECE distribution with various parameter values

\begin{tabular}{|c|c|c|c|c|c|}
\hline & & & $\theta=2$ & $\theta=3$ & $\theta=4$ \\
\hline \multirow{9}{*}{$\mu=3$} & \multirow[t]{3}{*}{$\gamma=0.5$} & $\tau=-2$ & 0.68 & 0.64 & 0.92 \\
\hline & & $\tau=1.5$ & 0.26 & 0.40 & 0.42 \\
\hline & & $\tau=5$ & 0.14 & 0.18 & 0.25 \\
\hline & \multirow[t]{3}{*}{$\gamma=1.5$} & $\tau=-2$ & 1.10 & 1.20 & 1.29 \\
\hline & & $\tau=1.5$ & 0.48 & 0.55 & 0.62 \\
\hline & & $\tau=5$ & 0.22 & 0.29 & 0.36 \\
\hline & \multirow[t]{3}{*}{$\gamma=5$} & $\tau=-2$ & 1.50 & 1.71 & 1.80 \\
\hline & & $\tau=1.5$ & 0.72 & 0.85 & 0.96 \\
\hline & & $\tau=5$ & 0.32 & 0.41 & 0.94 \\
\hline \multirow{9}{*}{$\mu=5$} & \multirow[t]{3}{*}{$\gamma=0.5$} & $\tau=-2$ & 0.14 & 0.24 & 0.24 \\
\hline & & $\tau=1.5$ & 0.12 & 0.18 & 0.17 \\
\hline & & $\tau=5$ & 0.06 & 0.11 & 0.14 \\
\hline & \multirow[t]{3}{*}{$\gamma=1.5$} & $\tau=-2$ & 0.65 & 0.74 & 0.81 \\
\hline & & $\tau=1.5$ & 0.27 & 0.33 & 0.33 \\
\hline & & $\tau=5$ & 0.13 & 0.18 & 0.22 \\
\hline & \multirow[t]{3}{*}{$\gamma=5$} & $\tau=-2$ & 0.95 & 0.95 & 1.01 \\
\hline & & $\tau=1.5$ & 0.42 & 0.51 & 0.62 \\
\hline & & $\tau=5$ & 0.19 & 0.25 & 0.31 \\
\hline \multirow{9}{*}{$\mu=7$} & \multirow[t]{3}{*}{$\gamma=0.5$} & $\tau=-2$ & 0.29 & 0.39 & 0.41 \\
\hline & & $\tau=1.5$ & 0.11 & 0.13 & 0.18 \\
\hline & & $\tau=5$ & 0.05 & 0.08 & 0.10 \\
\hline & \multirow[t]{3}{*}{$\gamma=0.5$} & $\tau=-2$ & 0.46 & 0.55 & 0.62 \\
\hline & & $\tau=1.5$ & 0.20 & 0.23 & 0.27 \\
\hline & & $\tau=5$ & 0.08 & 0.12 & 0.16 \\
\hline & \multirow[t]{3}{*}{$\gamma=5$} & $\tau=-2$ & 0.64 & 0.77 & 0.74 \\
\hline & & $\tau=1.5$ & 0.28 & 0.36 & 0.38 \\
\hline & & $\tau=5$ & 0.14 & 0.18 & 0.21 \\
\hline
\end{tabular}


Table 3: Variance of ECE distribution with various parameter values

\begin{tabular}{|c|c|c|c|c|c|}
\hline \multirow{9}{*}{$\mu=3$} & \multirow[t]{3}{*}{$\gamma=0.5$} & $\tau=-2$ & 0.39 & 0.21 & 0.20 \\
\hline & & $\tau=1.5$ & 0.08 & 0.09 & 0.06 \\
\hline & & $\tau=5$ & 0.01 & 0.02 & 0.02 \\
\hline & \multirow[t]{3}{*}{$\gamma=1.5$} & $\tau=-2$ & 0.16 & 0.25 & 0.17 \\
\hline & & $\tau=1.5$ & 0.10 & 0.07 & 0.12 \\
\hline & & $\tau=5$ & 0.01 & 0.02 & 0.02 \\
\hline & \multirow[t]{3}{*}{$\gamma=5$} & $\tau=-2$ & 0.11 & 0.21 & 0.20 \\
\hline & & $\tau=1.5$ & 0.10 & 0.12 & 0.14 \\
\hline & & $\tau=5$ & 0.02 & 0.01 & 0.17 \\
\hline \multirow{9}{*}{$\mu=5$} & \multirow[t]{3}{*}{$\gamma=5$} & $\tau=-2$ & 0.06 & 0.06 & 0.10 \\
\hline & & $\tau=1.5$ & 0.02 & 0.03 & 0.02 \\
\hline & & $\tau=5$ & 0.01 & 0.01 & 0.01 \\
\hline & \multirow[t]{3}{*}{$\gamma=1.5$} & $\tau=-2$ & 0.07 & 0.05 & 0.06 \\
\hline & & $\tau=1.5$ & 0.03 & 0.04 & 0.01 \\
\hline & & $\tau=5$ & 0.01 & 0.01 & 0.01 \\
\hline & \multirow[t]{3}{*}{$\gamma=5$} & $\tau=-2$ & 0.10 & 0.06 & 0.05 \\
\hline & & $\tau=1.5$ & 0.03 & 0.06 & 0.31 \\
\hline & & $\tau=5$ & 0.01 & 0.01 & 0.01 \\
\hline \multirow{9}{*}{$\mu=7$} & \multirow[t]{3}{*}{$\gamma=0.5$} & $\tau=-2$ & 0.03 & 0.06 & 0.05 \\
\hline & & $\tau=1.5$ & 0.02 & 0.01 & 0.02 \\
\hline & & $\tau=5$ & 0.01 & 0.01 & 0.01 \\
\hline & \multirow[t]{3}{*}{$\gamma=1.5$} & $\tau=-2$ & 0.03 & 0.06 & 0.06 \\
\hline & & $\tau=1.5$ & 0.02 & 0.02 & 0.01 \\
\hline & & $\tau=5$ & 0.01 & 0.01 & 0.01 \\
\hline & \multirow[t]{3}{*}{$\gamma=5$} & $\tau=-2$ & 0.02 & 0.07 & 0.03 \\
\hline & & $\tau=1.5$ & 0.01 & 0.02 & 0.02 \\
\hline & & $\tau=5$ & 0.01 & 0.01 & 0.01 \\
\hline
\end{tabular}


Table 4: Skewness of ECE distribution with various parameter values

\begin{tabular}{|c|c|c|c|c|c|}
\hline & & & $\theta=2$ & $\theta=3$ & $\theta=4$ \\
\hline \multirow{12}{*}{$\mu=3$} & \multirow[t]{3}{*}{$\gamma=0.5$} & $\tau=-2$ & 1.80 & 1.19 & 0.43 \\
\hline & & $\tau=1.5$ & 2.11 & 1.58 & 0.56 \\
\hline & & $\tau=5$ & 1.01 & 1.74 & 1.54 \\
\hline & \multirow[t]{3}{*}{$\gamma=1.5$} & $\tau=-2$ & 0.96 & 1.58 & 0.62 \\
\hline & & $\tau=1.5$ & 1.37 & 0.64 & 1.58 \\
\hline & & $\tau=5$ & 1.48 & 1.38 & 0.36 \\
\hline & \multirow[t]{3}{*}{$\gamma=5$} & $\tau=-2$ & 1.04 & 0.81 & 0.81 \\
\hline & & $\tau=1.5$ & 2.27 & 1.58 & 1.29 \\
\hline & & $\tau=5$ & 0.82 & 1.06 & 1.89 \\
\hline & \multirow[t]{3}{*}{$\gamma=0.5$} & $\tau=-2$ & 1.23 & 1.11 & 0.36 \\
\hline & & $\tau=1.5$ & 1.33 & 1.14 & 0.62 \\
\hline & & $\tau=5$ & 1.55 & 0.81 & 0.74 \\
\hline \multirow[t]{6}{*}{$\mu=5$} & \multirow[t]{3}{*}{$\gamma=1.5$} & $\tau=-2$ & 0.58 & 0.81 & 0.74 \\
\hline & & $\tau=1.5$ & 1.87 & 1.37 & 0.56 \\
\hline & & $\tau=5$ & 1.48 & 1.73 & 0.37 \\
\hline & \multirow[t]{3}{*}{$\gamma=5$} & $\tau=-2$ & 1.13 & 1.33 & 1.30 \\
\hline & & $\tau=1.5$ & 1.53 & 3.07 & 1.03 \\
\hline & & $\tau=5$ & 0.83 & 0.74 & 3.70 \\
\hline \multirow{9}{*}{$\mu=7$} & \multirow[t]{3}{*}{$\gamma=0.5$} & $\tau=-2$ & 0.67 & 0.44 & 0.42 \\
\hline & & $\tau=1.5$ & 2.58 & 1.10 & 1.26 \\
\hline & & $\tau=5$ & 1.63 & 0.69 & 1.74 \\
\hline & \multirow[t]{3}{*}{$\gamma=1.5$} & $\tau=-2$ & 0.81 & 1.82 & 1.32 \\
\hline & & $\tau=1.5$ & 1.46 & 1.10 & 1.51 \\
\hline & & $\tau=5$ & 1.74 & 0.86 & 1.05 \\
\hline & \multirow[t]{3}{*}{$\gamma=5$} & $\tau=-2$ & 0.67 & 1.23 & 0.32 \\
\hline & & $\tau=1.5$ & 0.14 & 1.27 & 1.41 \\
\hline & & $\tau=5$ & 1.12 & 0.38 & 0.25 \\
\hline
\end{tabular}


Table 5: Kurtosis of ECE distribution with various parameter values

\begin{tabular}{|c|c|c|c|c|c|}
\hline & & & $\theta=2$ & $\theta=3$ & $\theta=4$ \\
\hline \multirow{9}{*}{$\mu=3$} & \multirow[t]{3}{*}{$\gamma=0.5$} & $\tau=-2$ & 4.08 & 1.30 & -0.62 \\
\hline & & $\tau=1.5$ & 5.85 & 4.61 & -0.62 \\
\hline & & $\tau=5$ & 0.76 & 3.29 & 2.94 \\
\hline & \multirow[t]{3}{*}{$\gamma=1.5$} & $\tau=-2$ & 2.07 & 3.62 & 0.09 \\
\hline & & $\tau=1.5$ & 2.82 & 0.55 & 2.38 \\
\hline & & $\tau=5$ & 2.88 & 2.31 & -0.41 \\
\hline & \multirow[t]{3}{*}{$\gamma=5$} & $\tau=-2$ & 1.91 & 0.55 & 0.64 \\
\hline & & $\tau=1.5$ & 7.97 & 2.77 & 1.38 \\
\hline & & $\tau=5$ & 0.49 & 0.81 & 4.59 \\
\hline \multirow{9}{*}{$\mu=5$} & \multirow[t]{3}{*}{$\gamma=0.5$} & $\tau=-2$ & 1.22 & 2.08 & -0.31 \\
\hline & & $\tau=1.5$ & 0.90 & 2.01 & -0.31 \\
\hline & & $\tau=5$ & 2.01 & -0.16 & 2.30 \\
\hline & \multirow[t]{3}{*}{$\gamma=1.5$} & $\tau=-2$ & -0.04 & 1.69 & 0.39 \\
\hline & & $\tau=1.5$ & 3.49 & 1.46 & 0.07 \\
\hline & & $\tau=5$ & 4.18 & 4.43 & 0.16 \\
\hline & \multirow[t]{3}{*}{$\gamma=5$} & $\tau=-2$ & 0.64 & 1.73 & 1.51 \\
\hline & & $\tau=1.5$ & 3.80 & 14.42 & 0.31 \\
\hline & & $\tau=5$ & 0.30 & 1.02 & 19.18 \\
\hline \multirow{9}{*}{$\mu=7$} & \multirow[t]{3}{*}{$\gamma=0.5$} & $\tau=-2$ & 0.59 & -0.78 & -0.49 \\
\hline & & $\tau=1.5$ & 6.68 & 0.96 & 1.06 \\
\hline & & $\tau=5$ & 3.15 & -0.02 & 3.77 \\
\hline & \multirow[t]{3}{*}{$\gamma=1.5$} & $\tau=-2$ & 0.01 & 5.02 & 2.45 \\
\hline & & $\tau=1.5$ & 1.72 & 0.53 & 3.04 \\
\hline & & $\tau=5$ & 3.58 & 0.15 & 1.02 \\
\hline & \multirow[t]{3}{*}{$\gamma=5$} & $\tau=-2$ & -0.10 & 1.31 & -0.53 \\
\hline & & $\tau=1.5$ & -0.39 & 1.44 & 2.07 \\
\hline & & $\tau=5$ & 2.23 & -0.46 & -0.94 \\
\hline
\end{tabular}




\subsection{Renyi entropy}

In this section, the Renyi Entropy of the ECE distribution is discussed. The Rényi entropy tends to Shannon entropy when $\pi$ tends to 1 .

Using the expression in (35) and the pdf and cdf of the Exponential distribution, the Renyi entropy of the ECE distribution is derived as

$$
I_{R}(\pi)=\frac{1}{1-\pi} \log \int_{0}^{\infty} \sum_{j=0}^{\infty} \sum_{l=0}^{\infty} \sum_{k=0}^{\infty} \sum_{m=0}^{\infty} u_{h}\left(\mu e^{-\mu x}\right)^{\pi}\left(1-e^{-\mu x}\right)^{\theta(m+\pi)-\pi}
$$

Using the power series expansion,

$$
\left(1-e^{-\mu x}\right)^{\theta(m+\pi)-\pi}=\sum_{p=0}\left(\begin{array}{c}
\theta(m+\pi)-\pi \\
p
\end{array}\right) e^{-p \mu x}
$$

Therefore,

$$
I_{R}(\pi)=\frac{1}{1-\pi} \log \sum_{j=0}^{\infty} \sum_{l=0}^{\infty} \sum_{k=0}^{\infty} \sum_{m=0}^{\infty} \sum_{p=0}^{\infty} u_{h}(-1)^{p} \mu^{p}\left(\begin{array}{c}
\theta(m+\pi)-\pi \\
p
\end{array}\right) \int_{0}^{\infty} e^{-\mu x(p+1)} d x
$$

which finally gives

$$
I_{R}(\pi)=\frac{1}{1-\pi} \log \int_{0}^{\infty} \sum_{j=0}^{\infty} \sum_{l=0}^{\infty} \sum_{k=0}^{\infty} \sum_{m=0}^{\infty} \sum_{p=0}^{\infty} u_{h}(-1)^{p} \mu^{p}\left(\begin{array}{c}
\theta(m+\pi)-\pi \\
p
\end{array}\right) \frac{1}{\mu(p+1)} .
$$

\subsection{Order statistics}

Let $X_{1}, \ldots, X_{n}$ be a random sample from the Exponentiated Chen Exponential distribution, the pdf of $i^{\text {th }}$ order statistic, say $X_{i: n}^{E}$, can be written as

$f_{i: n}^{E}(x)=\frac{1}{B(i, n-i+1)} \sum_{v=0}^{n-1}(-1)^{v}\left(\begin{array}{c}n-i \\ v\end{array}\right) \sum_{j=0}^{\infty} \sum_{k=0}^{\infty} \sum_{l=0}^{\infty} \sum_{m=0}^{\infty} C_{h, i+v-1} \theta(m+1)\left(\mu e^{-\mu x}\right)\left(1-e^{-\mu x}\right)^{\theta(m+1)-1}$

Therefore, the first order $X_{1}$ of the pdf of the ECG distribution is

$$
f_{1: n}^{E}(x)=n \sum_{v=0}^{n-1}(-1)^{v}\left(\begin{array}{c}
n-1 \\
v
\end{array}\right) \sum_{j=0}^{\infty} \sum_{k=0}^{\infty} \sum_{l=0}^{\infty} \sum_{m=0}^{\infty} C_{h, j} \theta(m+1)\left(\mu e^{-\mu x}\right)\left(1-e^{-\mu x}\right)^{\theta(m+1)-1}
$$

and the last order $X_{n}$ as

$$
f_{n: n}^{E}(x)=n \sum_{v=0}^{n-1}(-1)^{v}\left(\begin{array}{c}
n-i \\
v
\end{array}\right) \sum_{j=0}^{\infty} \sum_{k=0}^{\infty} \sum_{l=0}^{\infty} \sum_{m=0}^{\infty} C_{h, n+v-1} \theta(m+1)\left(\mu e^{-\mu x}\right)\left(1-e^{-\mu x}\right)^{\theta(m+1)-1} .
$$

\subsection{Maximum likelihood estimation}

In this section, the estimation of the ECE distribution is done under complete and censored observations. This is done using the maximum likelihood estimation method. Let $X_{i}, \ldots, X_{n}$ be a random sample from the Exponentiated Chen Exponential distribution with observed values $x_{i}, \ldots, x_{n}$ and $\chi=(\tau, \theta, \gamma, \mu)$ be parameter vector. Then Log-Likelihood function of Exponentiated Chen Exponential Distribution under complete observation is obtained as

$l\left(x_{i} ; \tau, \theta, \gamma, \beta\right)=l=n \ln \gamma+n \gamma \ln A+n \ln \tau+n \ln \theta+\sum_{i=1}^{n} \ln \left(\mu e^{-\mu x_{i}}\right)+(\theta-1) \sum_{i=1}^{n} \ln \left(1-e^{-\mu x_{i}}\right)$ 


$$
+\sum_{i=1}^{n} \ln \left(1-e^{-\mu x_{i}}\right)^{\theta}+\sum_{i=1}^{n} \tau\left(1-e^{\left(1-e^{-\mu x_{i}}\right)^{\theta}}\right)+(\gamma-1) \sum_{i=1}^{n} \ln \left(1-e^{\tau\left(1-e^{\left(1-e^{-\mu x_{i}}\right)^{\theta}}\right)}\right)
$$

Differentiating the loglikelihood function with respect to $\chi$ gives

$$
\begin{aligned}
& \frac{\partial l}{\partial \gamma}=\frac{n}{\gamma}+\ln A+\ln \left(1-e^{\tau\left(1-e^{\left(1-e^{\left.-\mu x_{i}\right)^{\theta}}\right.}\right)}\right) \\
& \frac{\partial l}{\partial \tau}=\frac{n}{\tau}+n \gamma \frac{A^{\prime}}{A}+\sum_{i=1}^{n}\left(1-e^{-\mu x_{i}}\right)^{\theta}+(\gamma-1) \sum_{i=1}^{n} \frac{\left.\left(e^{\left(1-e^{-\mu x_{i}}\right)^{\theta}}-1\right) e^{\tau\left(1-e^{\left(1-e^{-\mu x_{i}}\right)^{\theta}}\right.}\right)}{1-e^{\tau\left(1-e^{\left(1-e^{-\mu x_{i}}\right)^{\theta}}\right)}} \\
& \frac{\partial l}{\partial \theta}=\frac{n}{\theta}+\sum_{i=1}^{n} \ln \left(1-e^{-\mu x_{i}}\right)+\sum_{i=1}^{n} \frac{\left(1-e^{-\mu x_{i}}\right)^{\theta} \ln \left(1-e^{-\mu x_{i}}\right)}{\left(1-e^{-\mu x_{i}}\right)^{\theta}} \\
& +(\gamma-1) \sum_{i=1}^{n} \frac{\tau\left(1-e^{-\mu x_{i}}\right)^{\theta} \ln \left(1-e^{-\mu x_{i}}\right) e^{\left(1-e^{-\mu x_{i}}\right)^{\theta}} e^{\tau\left(1-e^{\left(1-e^{-\mu x_{i}}\right)^{\theta}}\right)}}{1-e^{\tau\left(1-e^{\left(1-e^{\left.-\mu x_{i}\right)^{\theta}}\right.}\right)}} \\
& \frac{\partial l}{\partial \mu}=\sum_{i=1}^{n} \frac{e^{-\mu x}\left(1-\mu^{2}\right)}{\left(\mu e^{-\mu x}\right)}+(\theta-1) \sum_{i=1}^{n} \frac{\left(x e^{-\mu x}\right)}{\left(1-e^{-\mu x}\right)}+\sum_{i=1}^{n} \frac{\left.\theta\left(1-e^{-\mu x}\right)^{\theta-1}\left(x e^{-\mu x}\right)\right)}{\left(1-e^{-\mu x}\right)^{\theta}} \\
& \left.+\sum_{i=1}^{n} \tau\left(x_{i} e^{-\mu x_{i}}\right)\right)\left(1-e^{-\mu x_{i}}\right)^{\theta-1} e^{\left(1-e^{-\mu x_{i}}\right)^{\theta}}+(\gamma-1) \sum_{i=1}^{n} \frac{\left(x_{i} e^{-\mu x_{i}}\right)\left(1-e^{-\mu x_{i}}\right)^{\theta-1} e^{\left(1-e^{-\mu x_{i}}\right)^{\theta}}}{1-e^{\tau\left(1-e^{\left(1-e^{-\mu x_{i}}\right)^{\theta}}\right)}}
\end{aligned}
$$

where $A=\frac{1}{1-e^{\tau(1-e)}}$ and $\mathrm{A}^{\prime}=\frac{e^{\tau(1-e)}(1-e)}{\left(1-e^{\tau(1-e)}\right)^{2}}$.

Solving the non-linear equations in $(70),(71),(72)$, and (73) by equating to zero, the maximum likelihood estimates of the distribution are obtained. These equations can be solved numerically by using an iterative method, such as the Newton-Raphson technique, with the aid of statistical software.

For the censored observations, the $\log$ likelihood $\mathrm{L}(\zeta)^{*}$ function of the ECE distribution is

$$
\begin{gathered}
l\left(x_{i}, x_{i}^{+} ; \zeta^{*}\right)=l^{*}=r \ln \gamma+n \gamma \ln A+r \ln \tau+r \ln \theta+\sum_{i=1}^{r} \ln \left(e^{-\mu x_{i}}\right)+(\theta-1) \sum_{i=1}^{r} \ln \left(1-e^{-\mu x_{i}}\right) \\
+\sum_{i=1}^{r} \ln \left(1-e^{-\mu x_{i}}\right)^{\theta}+\sum_{i=1}^{r} \tau\left(1-e^{\left(1-e^{-\mu x}\right)^{\theta}}\right)+(\gamma-1) \sum_{i=1}^{r} \ln \left(1-e^{\tau\left(1-e^{\left(1-e^{-\mu x}\right)^{\theta}}\right)}\right) \\
+\sum_{i=r+1}^{n} \ln \left(1-\left(A\left[1-e^{\tau\left(1-e^{\left(1-e^{-\mu x^{+}}\right)^{\theta}}\right)}\right]\right)^{\gamma}\right)
\end{gathered}
$$

Differentiating $l^{*}$ with respect to the model parameters give

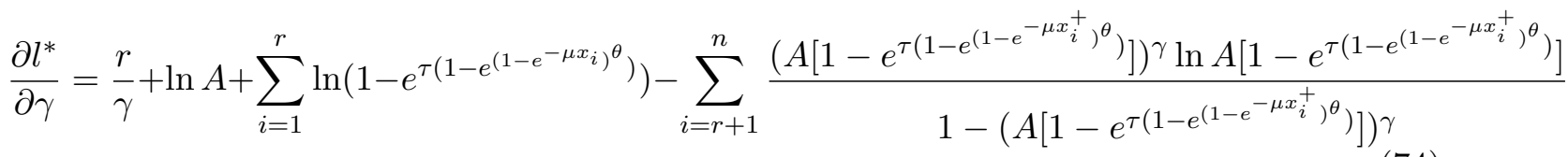

$$
\begin{aligned}
& \frac{\partial l}{\partial \tau}=\frac{r}{\tau}+n \gamma \frac{A^{\prime}}{A}+\sum_{i=1}^{r}\left(1-e^{\left(1-e^{-\mu x_{i}}\right)^{\theta}}\right)+(\gamma-1) \sum_{i=1}^{r} \frac{\left(e^{\left(1-e^{-\mu x_{i}}\right)^{\theta}}-1\right) e^{\tau\left(1-e^{\left(1-e^{\left.-\mu x_{i}\right)^{\theta}}\right.}\right)}-}{1-e^{\tau\left(1-e^{c^{\theta}}\right)}}- \\
& \sum_{i=r+1}^{n} \frac{\gamma\left(A^{\prime}\left(1-e^{\left(1-e^{-\mu x_{i}^{+}}\right)^{\theta}}\right)+A\left(\left(e^{\left(1-e^{-\mu x_{i}^{+}}\right)^{\theta}}-1\right) e^{\tau\left(1-e^{\left(1-e^{-\mu x_{i}^{+}}\right)^{\theta}}\right)}\right)\right)^{\gamma-1}}{1-\left(A\left[1-e^{\tau\left(1-e^{\left(1-e^{-\mu x_{i}^{+}}\right)^{\theta}}\right)}\right]\right)^{\gamma}}
\end{aligned}
$$




$$
\begin{aligned}
& \frac{\partial l}{\partial \theta}=\frac{r}{\theta}+\sum_{i=1}^{r} \ln \left(1-e^{-\mu x_{i}}\right)+\sum_{i=1}^{r} \frac{\left(1-e^{-\mu x_{i}}\right)^{\theta} \ln \left(1-e^{-\mu x_{i}}\right)}{\left(1-e^{-\mu x_{i}}\right)^{\theta}}
\end{aligned}
$$

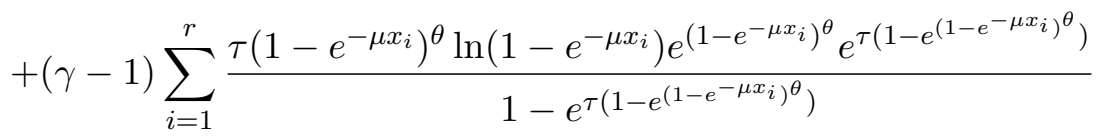

$$
\begin{aligned}
& -\sum_{i=r+1}^{n} \frac{\gamma A^{\gamma} \tau\left(1-e^{-\mu x_{i}^{+}}\right)^{\theta} \ln \left(1-e^{-\mu x_{i}^{+}}\right) e^{\tau\left(1-e^{\left(1-e^{-\mu x_{i}^{+}}\right)^{\theta}}\right)}\left(1-e^{\tau\left(1-e^{\left.\left(1-e^{-\mu x_{i}^{+}}\right)^{\theta}\right)}\right)^{\gamma-1}}\right.}{1-\left(A\left[1-e^{\tau\left(1-e^{\left(1-e^{-\mu x_{i}^{+}}\right)^{\theta}}\right)}\right]\right)^{\gamma}} \\
& \frac{\partial l^{*}}{\partial \mu}=\sum_{i=1}^{r} \frac{e^{-\mu x_{i}}\left(1-\mu^{2}\right)}{\mu e^{-\mu x_{i}}}+(\theta-1) \sum_{i=1}^{r} \frac{x_{i} e^{-\mu x_{i}}}{1-e^{-\mu x_{i}}}+\sum_{i=1}^{r} \theta\left(1-e^{-\mu x_{i}}\right)^{\theta-1}\left(x_{i} e^{-\mu x_{i}}\right)
\end{aligned}
$$

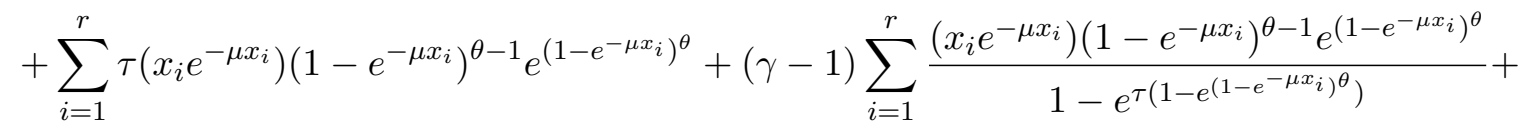

$$
\begin{aligned}
& \sum_{i=r+1}^{n} \frac{\gamma \tau\left(A\left(1-e^{\tau\left(1-e^{\left(1-e^{-\mu x_{i}^{+}}\right)^{\theta}}\right)}\right)\right)^{\gamma-1} e^{\tau\left(1-e^{\left(1-e^{-\mu x_{i}^{+}}\right)^{\theta}}\right)} e^{\left(1-e^{-\mu x_{i}^{+}}\right)^{\theta} \theta\left(1-e^{-\mu x_{i}^{+}}\right)^{(\theta-1)}\left(x_{i}^{+} e^{-\mu x_{i}^{+}}\right)}}{1-\left(A\left[1-e^{\tau\left(1-e^{\left(1-e^{-\mu x_{i}^{+}}\right)^{\theta}}\right)}\right]\right)^{\gamma}}
\end{aligned}
$$

Solving equations $(74),(75),(76),(77)$ equal zero gives the maximum likelihood estimates of the ECE distribution. This is done iteratively with the aid of statistical software.

\subsection{Quantile function}

The quantile function $q_{E C E}(u)$ of the ECE distribution is given as

$$
q_{E C E}(u)=\frac{-\ln \left\{1-\left[\left(\ln \left(1-\left(\frac{\ln \left(1-\frac{u^{\frac{1}{g}}}{A}\right)}{\tau}\right)\right)\right)^{\frac{1}{\theta}}\right]\right\}}{\mu}
$$

where $\mathrm{u}$ is distributed to Uniform $(0,1)$.

\subsection{Application to real datasets}

In this section, the ECE distribution is applied to three real datasets to illustrate the fitting of the ECG family of distributions. The ECE distribution is compared with the fittings of its submodels and other competitive distributions. The pdf of the submodels in literature are in Table 1. The competitive models considered are the Beta Exponential distribution (Nadarajah and Kotz 2006) and the Exponentiated Generalized Exponential distribution (Okagbue, Oguntunde, Ugwoke, Opanuga, and Erondu 2017).

\section{Nicotine dataset}

The dataset contains 346 Nicotine measurements made from brands of cigarettes as extracted from Handique and Chakraborty (2016) The summary of the Nicotine dataset is presented in Table 6. Fitting the distributions considered for this data, the histogram with their respective fitting is revealed in Figure 3. The TTT plot of the data in Figure 4 shows that the data has a non-decreasing hazard rate. After analysis, Table 7 shows the results of the MLEs and their respective standard errors. Using the model comparison criterion, it clearly shows that the ECE distribution performs better than its submodels and relative distributions. This is due to the lowest values of the AIC, BIC, CAIC, HQIC, $\mathrm{A}^{*}, \mathrm{~W}^{*}$ and the highest p-value of the KS test. 
Table 6: Summary statistics of Nicotine data

\begin{tabular}{|llllll|}
\hline Minimum & First Quartile & Median & Mean & Third Quartile & Maximum \\
\hline 0.1000 & 0.6000 & 0.9000 & 0.8526 & 1.1000 & 2.0000 \\
\hline
\end{tabular}

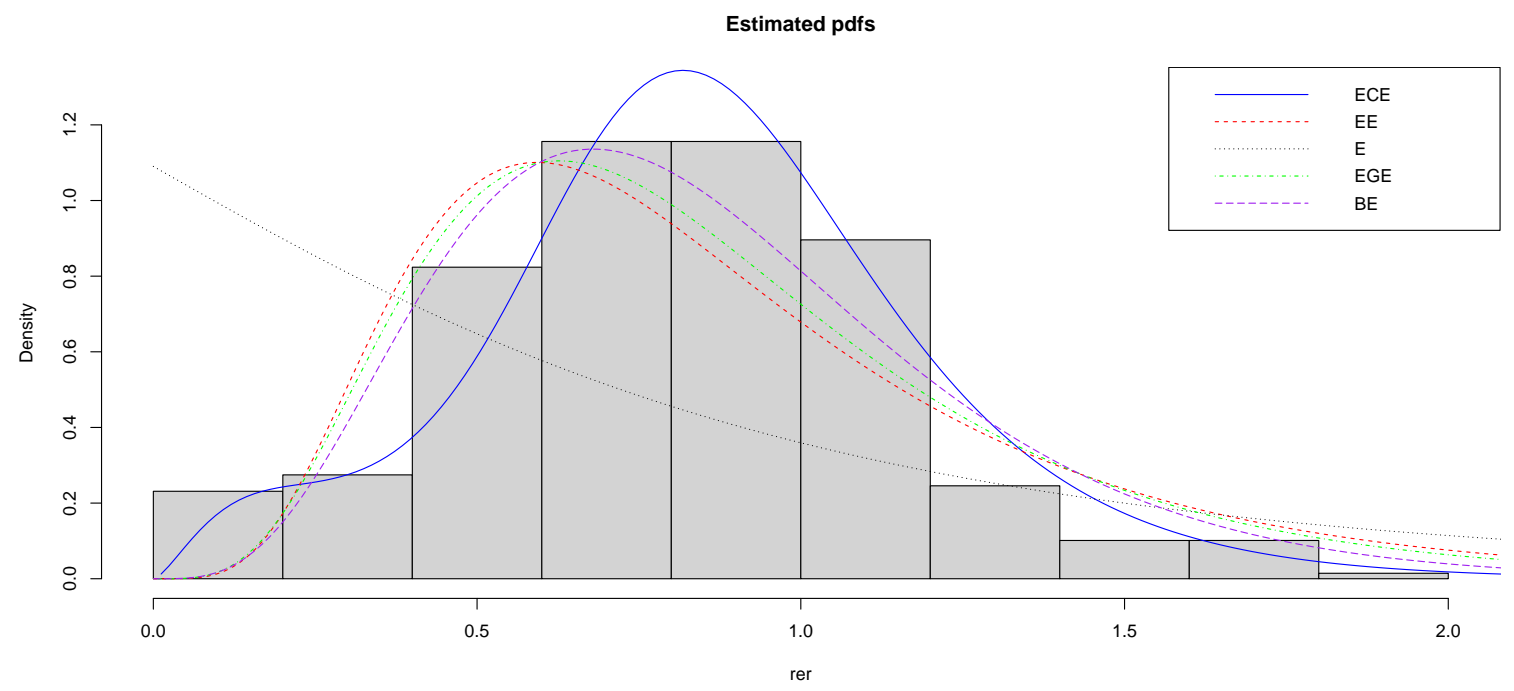

Figure 3: Observed histogram and fitted densities of the ECE distribution and other models for the Nicotine Data

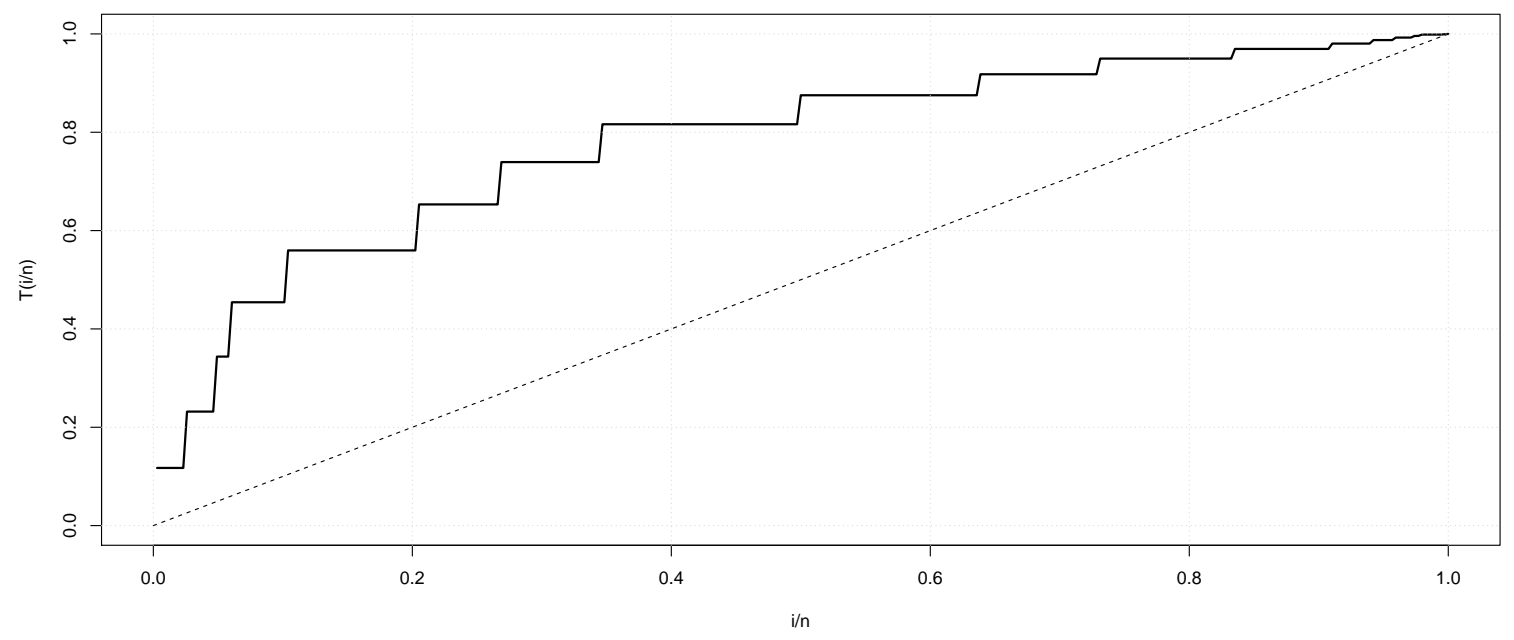

Figure 4: TTT plots for the Nicotine data

\section{Windshield data set}

The windshield on a large aircraft is a complex piece of equipment, comprised basically of several layers of material, including a very strong outer skin with a heated layer just beneath it, all laminated under high temperature and pressure. Failures of these items are not structural failures. Instead, they typically involve damage or delamination of the nonstructural outer ply or failure of the heating system. These failures do not result in damage to the aircraft but do result in the replacement of the windshield. The data on failure and service times for a particular model windshield given in Table 16.11 of Murthy, Xie, and Jiang (2004) is considered. The data consists of 153 observations, of which 88 are classified as failed 
Table 7: MLE and goodness of fits for Nicotine data

\begin{tabular}{|llllllllllll|}
\hline Model & & Est & S.E & -L & AIC & BIC & CAIC & HQIC & A $^{*}$ & W $^{*}$ & KS \\
\hline ECE & $\gamma$ & 0.5516 & 0.0735 & 107.04 & 222.07 & 237.46 & 222.19 & 228.21 & 0.38 & 2.09 & $0.098(0.003)$ \\
& $\tau$ & -2.688 & 1.536 & & & & & & & & \\
& $\theta$ & 6.827 & 3.017 & & & & & & & & \\
& $\mu$ & 4.119 & 0.253 & & & & & & & & \\
EE & $\gamma$ & 5.526 & 0.414 & 149.22 & 302.44 & 310.14 & 302.47 & 305.52 & 9.42 & 1.59 & $0.22(0.23)$ \\
& $\mu$ & 2.726 & 0.128 & & & & & & & & \\
E & $\mu$ & 1.173 & 0.063 & 289.83 & 583.66 & 587.51 & 583.67 & 585.20 & 58.38 & 11.79 & $0.24(0.17)$ \\
EGE & $\gamma$ & 0.828 & 0.089 & & 304.44 & 315.99 & 304.51 & 309.06 & 9.41 & 1.60 & $0.22(0.23)$ \\
& $\phi$ & 5.527 & 0.514 & & & & & & & & \\
BE & $\gamma$ & 4.922 & 0.364 & 135.02 & 276.04 & 287.59 & 276.11 & 280.66 & 6.48 & 1.09 & $0.24(0.16)$ \\
& $\beta$ & 17.433 & 8.216 & & & & & & & & \\
& $\phi$ & 0.298 & 0.128 & & & & & & & & \\
\hline
\end{tabular}

windshields, and the remaining 65 are service times of windshields that had not failed at the time of observation. The unit for measurement is $1000 \mathrm{~h}$. Fitting the distributions considered for this data, the histogram with their respective fitting is revealed in Figure 5. The TTT plot of the data in Figure 6 shows that the data has a non-decreasing hazard rate. After analysis, Table 9 shows the results of the MLEs and their respective standard errors. Using the model comparison criterion, it clearly shows that the ECE distribution performs better than its submodels and relative distributions. This is due to the lowest values of the AIC, BIC, CAIC, HQIC, $\mathrm{A}^{*}, \mathrm{~W}^{*}$ and the highest p-value of the $\mathrm{KS}$ test.

Table 8: Summary statistics of Windshield data

\begin{tabular}{|llllll|}
\hline Minimum & First Quartile & Median & Mean & Third Quartile & Maximum \\
\hline 0.040 & 1.866 & 2.385 & 2.563 & 3.376 & 4.663 \\
\hline
\end{tabular}

Table 9: MLE and goodness of fits for failure times of Windshield data

\begin{tabular}{|llllllllllll|}
\hline Model & & Est & S.E & -L & AIC & BIC & CAIC & HQIC & A* & W & KS \\
\hline ECE & $\gamma$ & 0.244 & 0.16 & 128.82 & 265.64 & 266.14 & 275.41 & 269.57 & 0.48 & 0.064 & $0.06(0.82)$ \\
& $\tau$ & -7.98 & 4.98 & & & & & & & & \\
& $\theta$ & 3.48 & 1.92 & & & & & & & & \\
& $\mu$ & 1.27 & 0.14 & & & & & & & & \\
EE & $\gamma$ & 3.66 & 0.67 & 141.17 & 292.34 & 292.50 & 297.24 & 294.32 & 2.11 & 0.27 & $0.13(0.12)$ \\
& $\mu$ & 0.62 & 0.07 & & & & & & & & \\
E & $\mu$ & 0.29 & 0.03 & 165.91 & 333.82 & 333.87 & 336.27 & 334.80 & 1.50 & 0.18 & $0.29(0.17)$ \\
EGE & $\gamma$ & 1.89 & 210.34 & 141.39 & 288.79 & 288.09 & 296.12 & 291.74 & 4.88 & 0.76 & $0.53(0.00)$ \\
& $\phi$ & 1.89 & 210.34 & & & & & & & & \\
& $\mu$ & 0.76 & 0.08 & & & & & & & & \\
BE & $\gamma$ & 3.51 & 0.52 & 138.44 & 282.22 & 283.18 & 290.21 & 285.83 & 9.76 & 1.73 & $0.99(0.00)$ \\
& $\beta$ & 14.19 & 15.10 & & & & & & & & \\
& $\phi$ & 0.09 & 0.09 & & & & & & & & \\
\hline
\end{tabular}




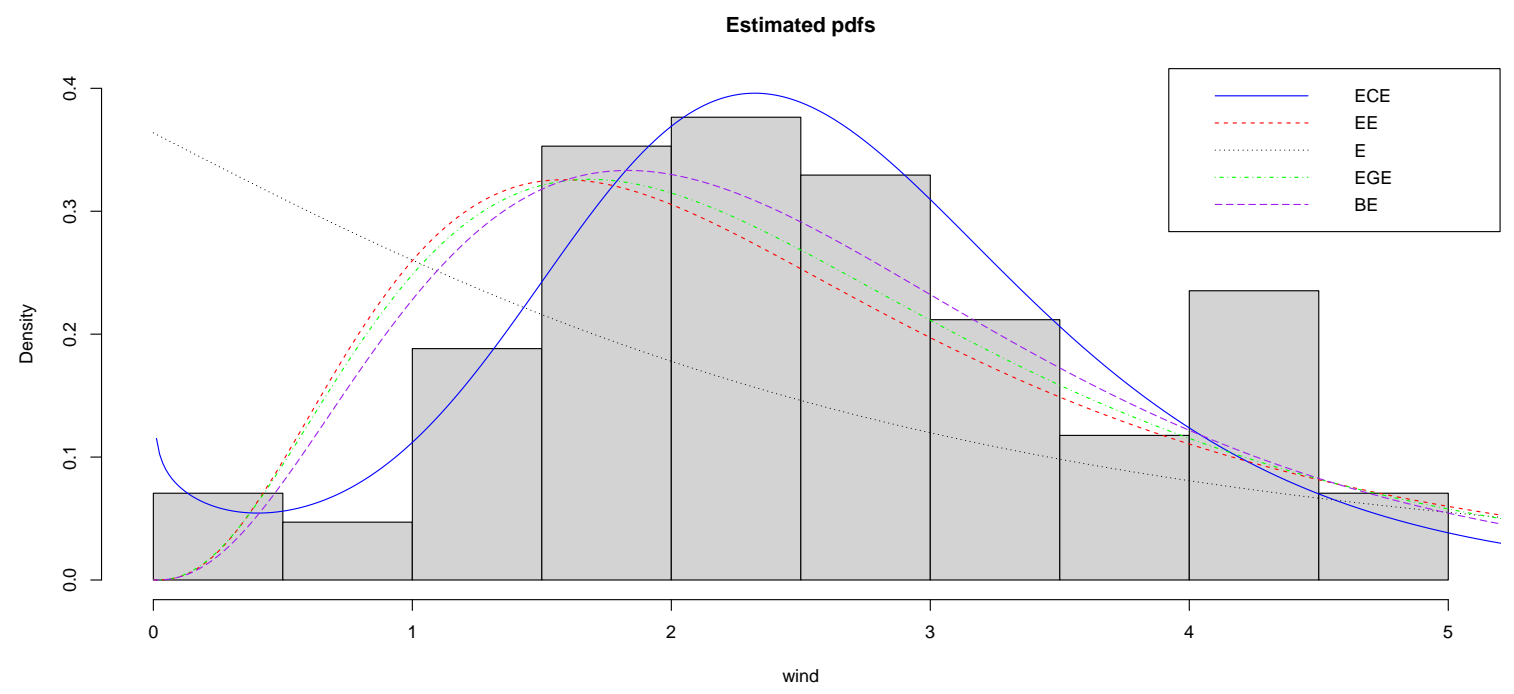

Figure 5: Observed histogram and fitted densities of the ECE distribution and other models for the Failure times of Windshield

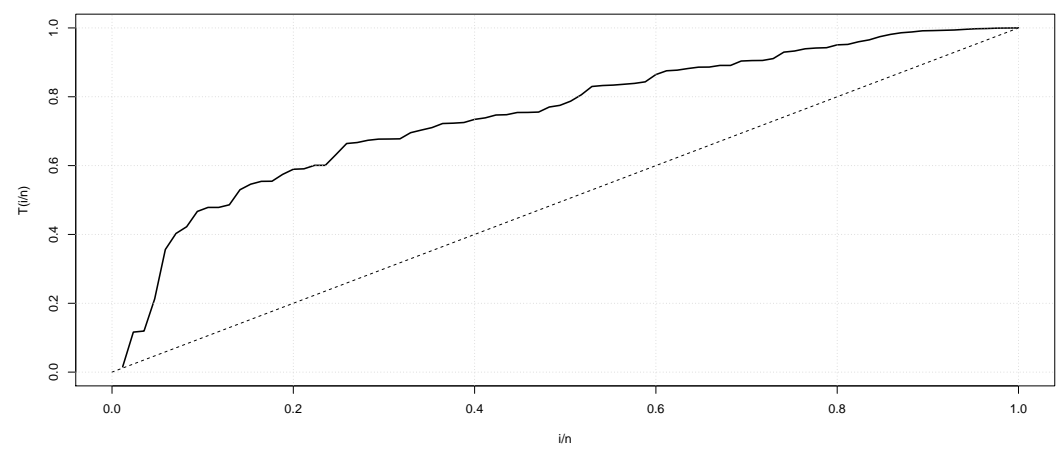

Figure 6: TTT plot for the Failure times of Windshield

\section{Conclusion}

In this work, the Exponentiated Chen-G family of distributions was studied. The mixture representation of the family was derived, which enables us to study some statistical properties such as moments, moment generating function, order statistics, entropy, reliability functions, and quantile function. The estimation of the parameters of the model under complete and censored observation has also been discussed. Finally, applications of the ECG family distribution were done to two real datasets in which the ECG family performs better than its subfamilies. 


\section{References}

Afify A, Cordeiro G, Yousof HM, Alzaatreh A, Nofal Z (2016). "The Kumaraswamy Transmuted-G Family of Distributions: Properties and Applications." Journal of data science, 14(2), 245-270. doi:10.6339/JDS.201604_14(2).0004.

Afify A, Yousof HM, Nadarajah S (2017). "The Beta Transmuted-H Family for Lifetime Data." Statistics and Its Interface, 10, 505-520. doi:10.4310/SII.2017.v10.n3.a13.

Alizadeh M, Emadi M, Doostparast M, Cordeiro G, Ortega E, Pescim R (2015). "A New Family of Distributions: The Kumaraswamy Odd Log-Logistic, Properties and Applications." Hacettepe Journal of Mathematics and Statistics, Article in Press., pp. 1-23. doi: 10.15672/HJMS . 2014418153.

Alizadeh M, Rasekhi M, Yousof HM, Hamedani G (2017). "The Transmuted Weibull G Family of Distributions." Hacettepe University Bulletin of Natural Sciences and Engineering Series B: Mathematics and Statistics, 47, 1671-1689. doi:10.15672/HJMS.2017.440.

Anzagra L, Sarpong S, Nasiru S (2020). "Chen-G Class of Distributions." Cogent Mathematics Es Statistics, 7(1), 1721401. doi:10.1080/25742558.2020.1721401.

Awodutire P (2020). "Chen Pareto Distribution: Properties and Application." Pakistan Journal of Statistics and Operation Research, 16, 812-826. doi:10.18187/pjsor.v16i4. 3418.

Badr MM, Elbatal I, Jamal F, Chesneau C, Elgarhy M (2020). "The Transmuted Odd FréchetG Family of Distributions: Theory and Applications." Mathematics, 8(6). ISSN 2227-7390. URL https://www.mdpi.com/2227-7390/8/6/958.

Bourguignon M, Silva RB, M CG (2014). "The Weibull-G Family of Probability Distributions." Journal of Data Science, 12, 53-68.

Cordeiro G, Afify A, Yousof HM, Pescim R, Aryal G (2017). "The Exponentiated Weibull-H Family of Distributions: Theory and Applications." Mediterranean Journal of Mathematics, 14. doi:10.1007/s00009-017-0955-1.

Cordeiro GM, Ortega EMM, Popović BV, Pescim RR (2014). "The Lomax Generator of Distributions: Properties, Minification Process and Regression Model." Applied Mathematics and Computation, 247, 465-486. ISSN 0096-3003. doi:https://doi.org/10. 1016/j.amc.2014.09.004. URL https://www.sciencedirect.com/science/article/ pii/S009630031401220X.

Eugene N, Lee C, Famoye F (2002). "Beta-Normal Distribution and Its Applications." Communications in Statistics - Theory and Methods, 31(4), 497-512. doi:10.1081/ STA-120003130. https://doi.org/10.1081/STA-120003130, URL https://doi.org/ 10.1081/STA-120003130.

Gradshteyn IS, Ryzhik IM (2007). "Table of Integrals, Series, and Products." Academic Press, New York.

Gupta RC, Gupta PI, Gupta RD (1998). "Modeling Failure Time Data by Lehmann Alternatives." Communication in Statistics - Theory Methods, 27, 887-904.

Handique L, Chakraborty S (2016). "Beta Generated Kumaraswamy-G and Other New Families of Distributions." arXiv e-prints, arXiv:1603.00634.

Handique L, Chakraborty S, Hamedani G (2017). "The Marshall-Olkin-Kumaraswamy-G Family of Distributions." Journal of Statistical Theory and Applications, 16, 427-447. ISSN 2214-1766. doi:10.2991/jsta.2017.16.4.2. 
Ibrahim S, Abu-Zinadah H (2009). "Exponentiated Pareto Distribution: Different Method of Estimations." International Journal of Contemporary Mathematical Sciences, 4, 677-693.

Mudholkar GS, Srivastava DK (1993). "Exponentiated Weibull Family for Analyzing Bathtub Failure Data." IEEE Transactions on Reliability, 42(2), 299-302. doi:10.1109/24.229504.

Murthy DNP, Xie M, Jiang R (2004). "Weibull Models." Wiley.

Nadarajah S, Kotz S (2006). "The Beta Exponential Distribution." Reliability Engineering and System Safety, 91(6), 689-697.

Nofal ZM, Altun E, Afify ZA, Ahsanullah M (2019). "The Generalized Kumaraswamy-G Family of Distributions." Journal of Statistical Theory and Applications, 18, 329-342. ISSN 2214-1766. doi:https://doi.org/10.2991/jsta.d.191030.001.

Okagbue HI, Oguntunde PE, Ugwoke PO, Opanuga AA, Erondu EC (2017). "Exponentiated Generalized Exponential Distribution: Ordinary Differential Equations." Transactions on Engineering Technologies. WCECS 2017. doi:10.1007/978-981-13-2191-7_24.

Rodrigues J, Percontini A, Hamedani G (2017). "The Exponentiated Generalized Lindley Distribution." Asian Research Journal of Mathematics, 5, 1-14. doi:10.9734/ARJOM/ $2017 / 26892$.

Shaw WT, Buckley IR (2009). "The Alchemy of Probability Distributions: Beyond GramCharlier Expansions, and a Skew-Kurtotic-Normal Distribution from a Rank Transmutation Map." 0901.0434.

Tahir MH, Cordeiro GM, Alzaatreh A, Mansoor M, Zubair M (2016). "The Logistic-X Family of Distributions and Its Applications." Communications in Statistics - Theory and Methods, 45(24), 7326-7349. doi:10.1080/03610926.2014.980516.

Yousof HM, Afify A, Alizadeh M, Butt N, Hamedani G, Ali M (2015). "The Transmuted Exponentiated Generalized-G Family of Distributions." Pakistan Journal of Statistics and Operation Research, 11, 441-464. doi:10.18187/pjsor.v11i4.1164.

Yousof HM, Afify A, Nadarajah S, Hamedani G, Aryal G (2018). "The Marshall-Olkin Generalized-G Family of Distributions with Applications." Statistica, 78(3), 273-295. doi: 10.6092/issn.1973-2201/7662.

Yousof HM, Rasekhi M, Afify A, Ghosh I, Alizadeh M, Hamedani G (2017). "The Beta Weibull-G Family of Distributions: Theory, Characterizations and Applications." Pakistan Journal of Statistics, 33, 95-116. 


\section{Appendix}

The probability density function and cumulative density function in $\mathbf{R}$

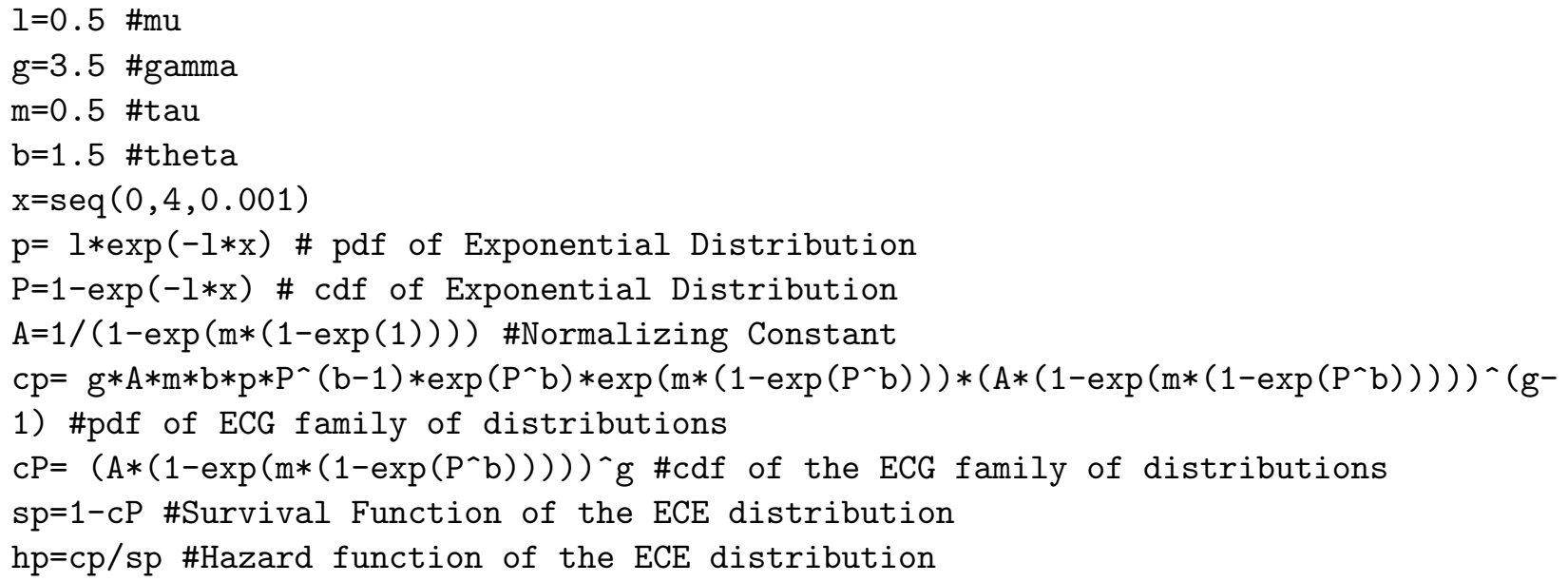

\section{Simulation of data, distributed to the ECE distribution}

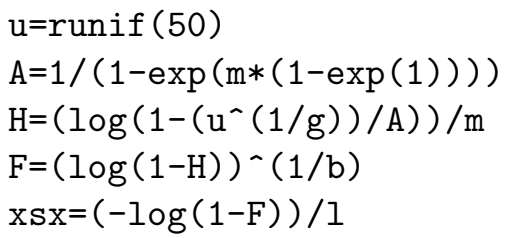

\section{Model fitting of ECE distribution and its submodels to Nicotine data using $\mathbf{R}$}

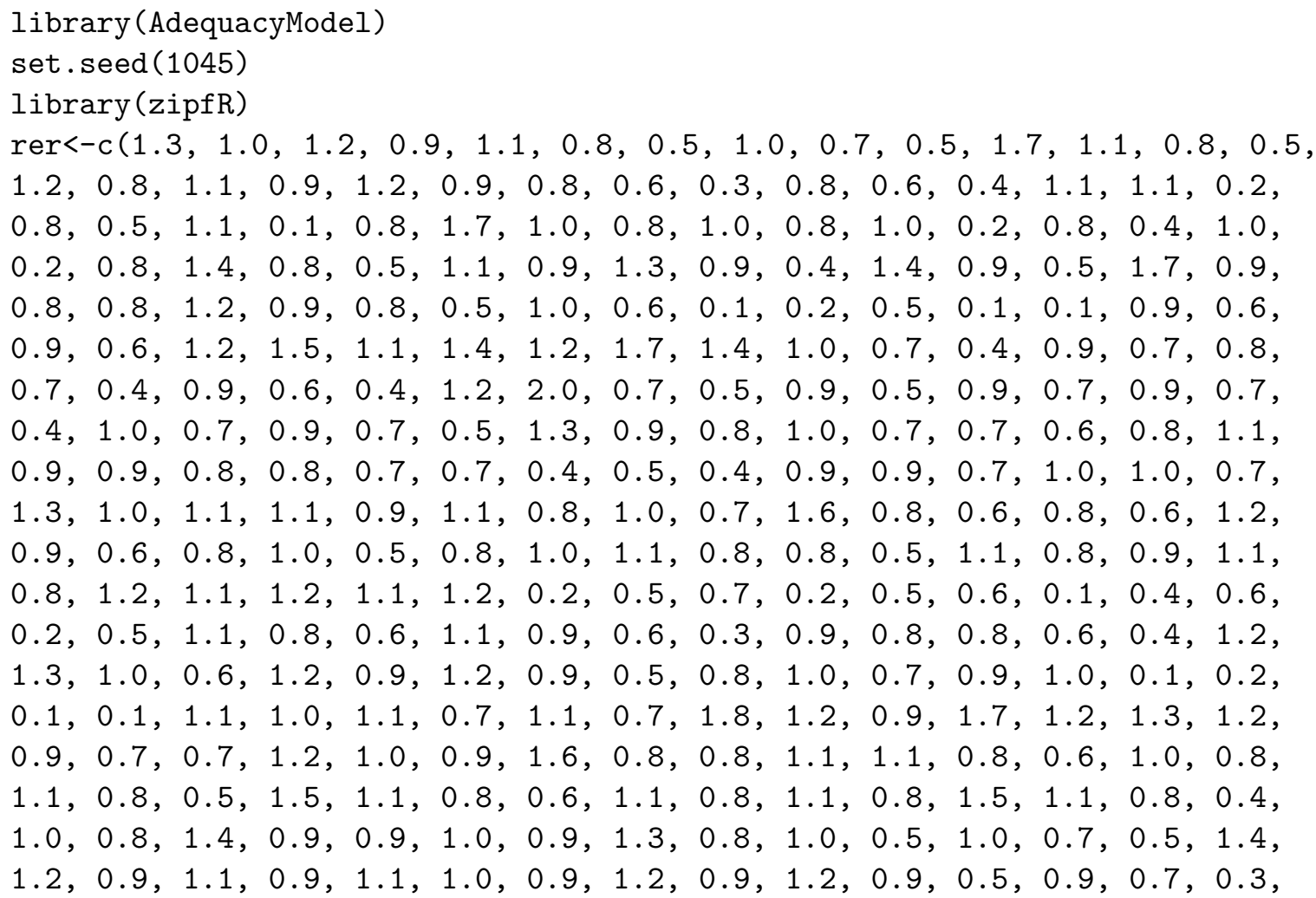


$1.0,0.6,1.0,0.9,1.0,1.1,0.8,0.5,1.1,0.8,1.2,0.8,0.5,1.5,1.5$, $1.0,0.8,1.0,0.5,1.7,0.3,0.6,0.6,0.4,0.5,0.5,0.7,0.4,0.5,0.8$, $0.5,1.3,0.9,1.3,0.9,0.5,1.2,0.9,1.1,0.9,0.5,0.7,0.5,1.1,1.1$, $0.5,0.8,0.6,1.2,0.8,0.4,1.3,0.8,0.5,1.2,0.7,0.5,0.9,1.3,0.8$, $1.2,0.9)$

\#negative loglikelihood function to estimate the parameters for CTTIG pdf <- function(params, $x$ )

\{

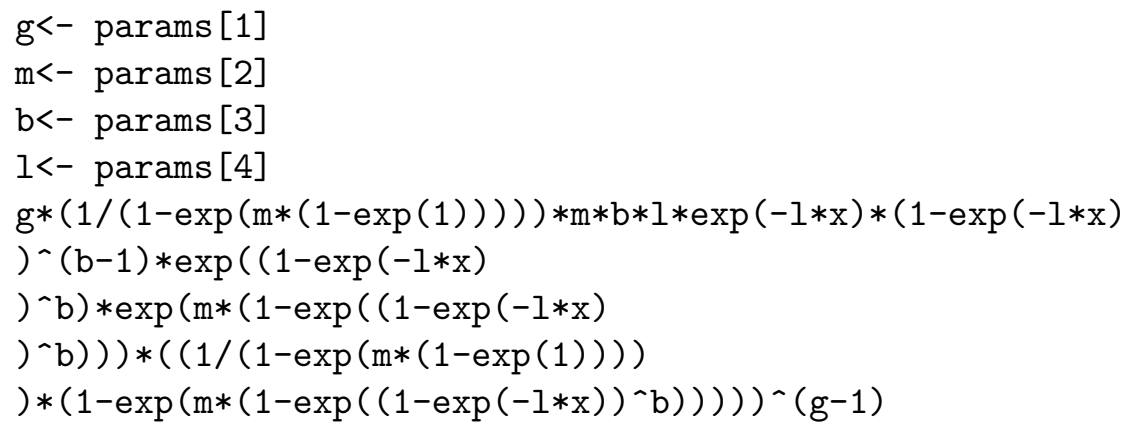


\}

result1 $=$ goodness.fit $(\operatorname{pdf}=\operatorname{pdf} 1, \operatorname{cdf}=\operatorname{cdf} 1$, starts $=c(1,1)$, data $=$ rer, method $=$ "BFGS", domain $=c(0$, Inf $), \mathrm{mle}=$ NULL, lim_inf $=c(0,0)$, $\lim _{-}$sup $=c(10,10), \mathrm{S}=250$, prop=0.1, N=50)

result1

\#E

\#negative loglikelihood function to estimate the parameters for CTTIG pdf $2<-$ function(params, $x$ )

\{

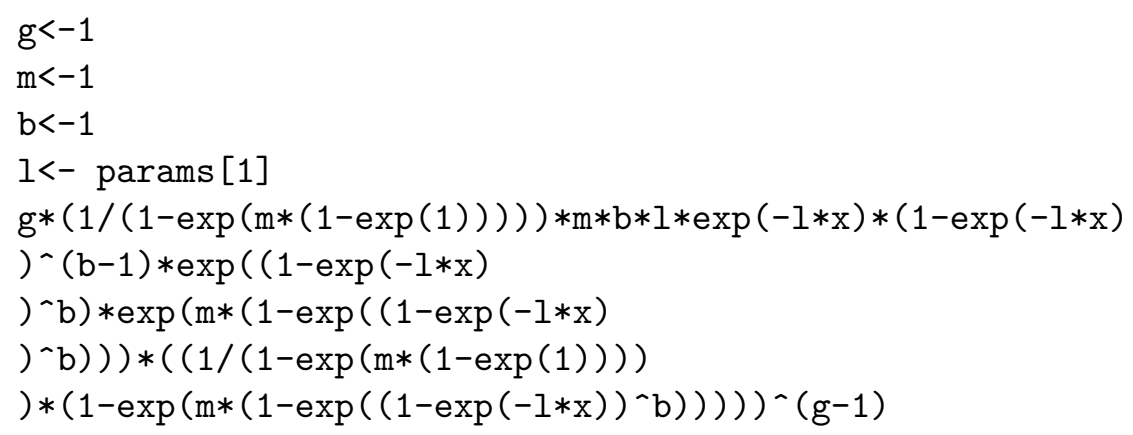




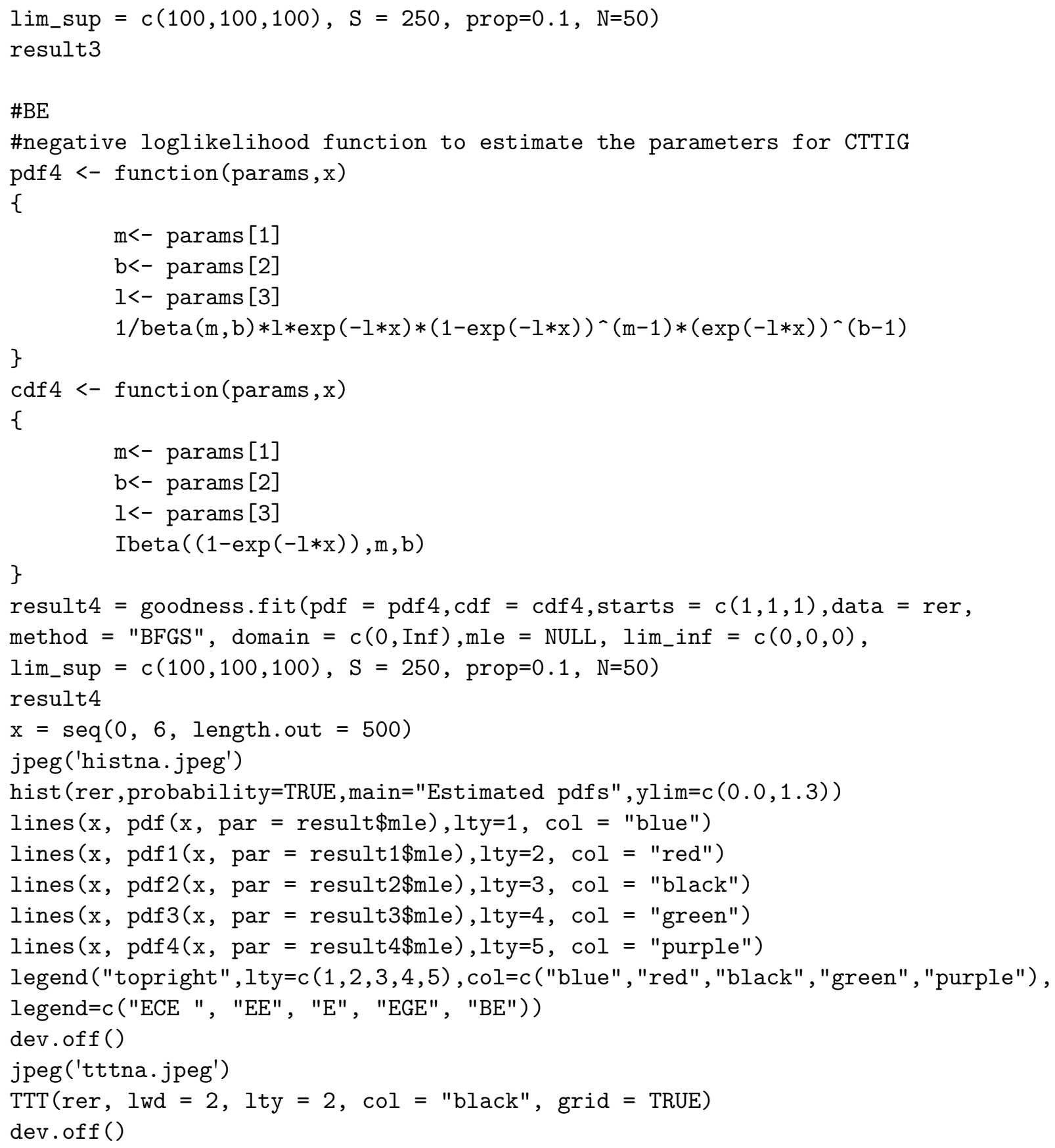

\section{Model fitting of ECE distribution and its submodels to Windscreen Data Using $\mathbf{R}$}

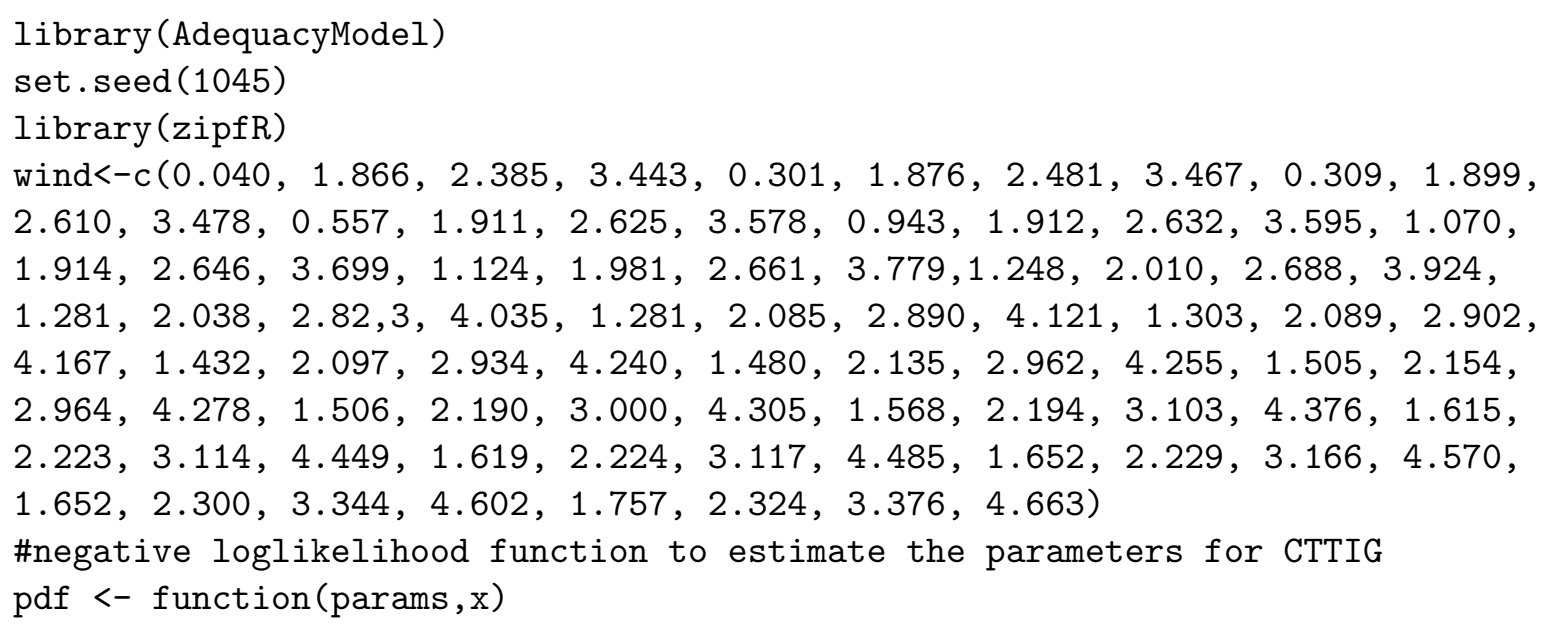


\{

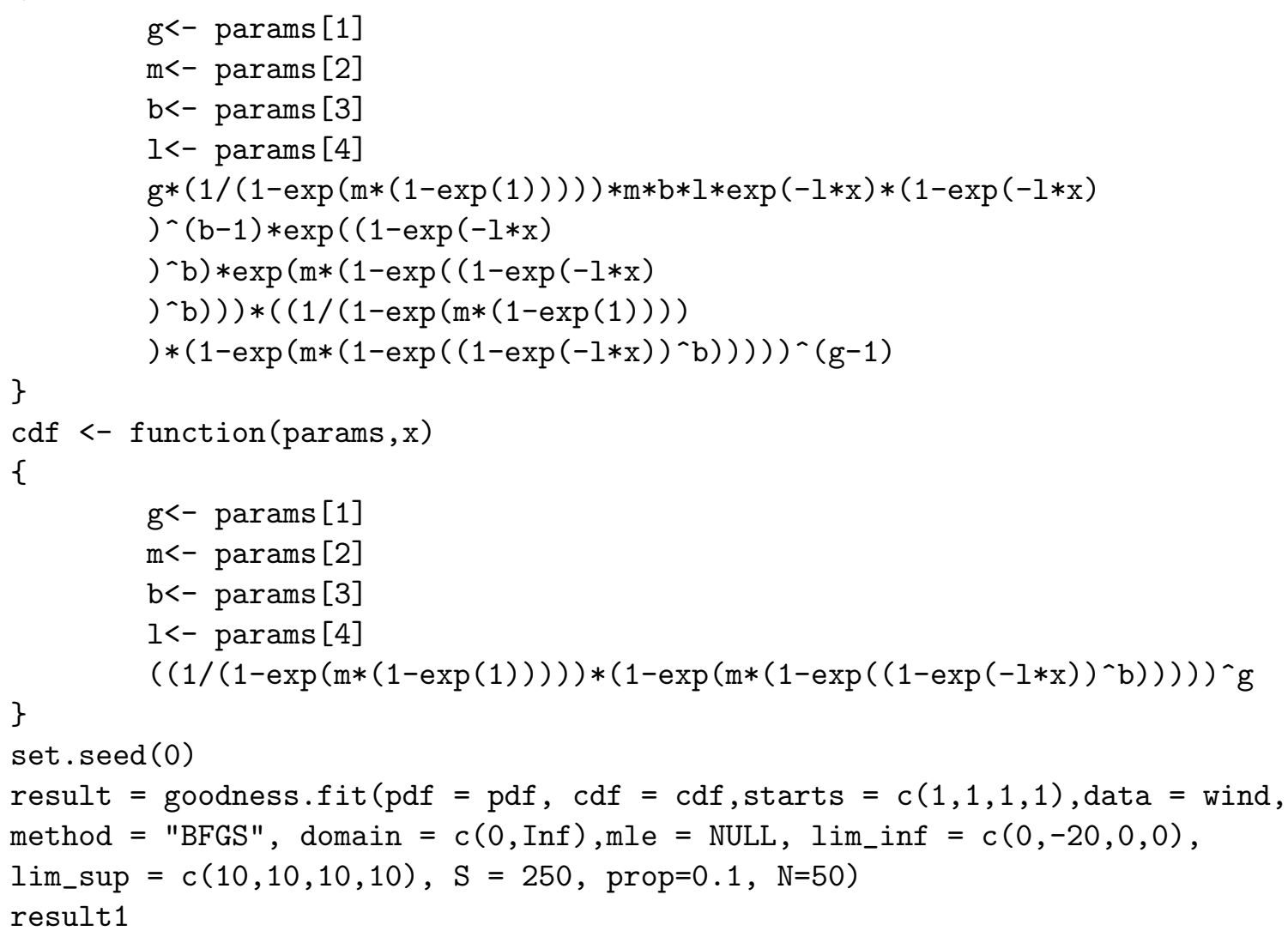


\#negative loglikelihood function to estimate the parameters for CTTIG pdf $2<-$ function (params, $x$ )

\{

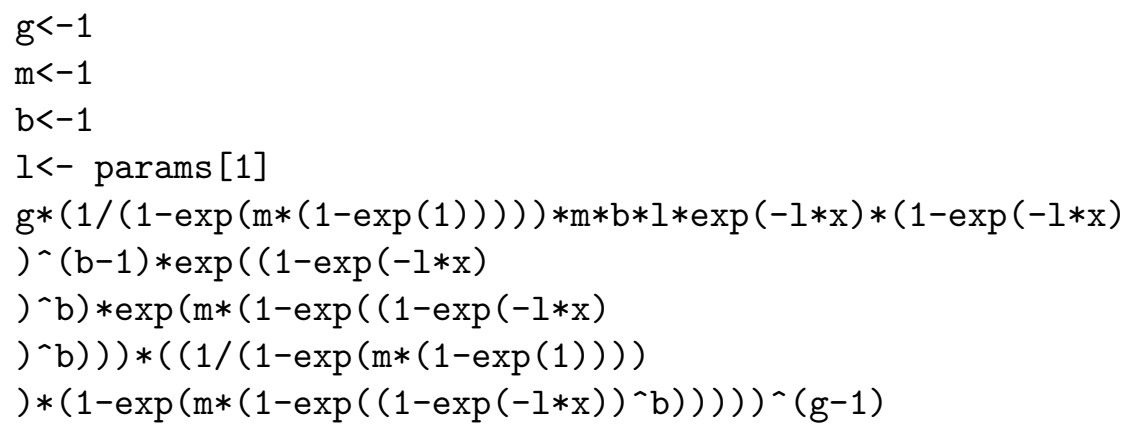

\}

cdf $2<-$ function (params, $x$ )

\{

$\mathrm{g}<-1$

$\mathrm{m}<-1$

$\mathrm{b}<-1$

$1<-\operatorname{params}[1]$

$\left((1 /(1-\exp (m *(1-\exp (1))))) *\left(1-\exp \left(m *\left(1-\exp \left((1-\exp (-1 * x))^{\wedge} b\right)\right)\right)\right)\right)^{\wedge} g$

\}

result2 $=$ goodness.fit (pdf $=\operatorname{pdf} 2, \operatorname{cdf}=\operatorname{cdf} 2$, starts $=c(1)$, data $=$ wind, method $=$ "BFGS", domain $=c(0$, Inf $), \mathrm{mle}=$ NULL, lim_inf $=c(0,0)$,

$\lim _{-} \sup =c(20), \mathrm{S}=250$, prop=0.1, $\left.\mathrm{N}=50\right)$

result2

\#EGE

\#negative loglikelihood function to estimate the parameters for CTTIG pdf3 <- function (params, $x$ )

\{

$\mathrm{g}<-$ params [1]

$\mathrm{m}<-$ params [2]

$\mathrm{b}<-1$

$1<-$ params [3]

$\mathrm{g} * \mathrm{l} *(1 / \operatorname{beta}(\mathrm{m}, \mathrm{b})) * \exp (-\mathrm{l} * \mathrm{x}) *(1-\exp (-\mathrm{l} * \mathrm{x})) \wedge(\mathrm{g} * \mathrm{~m}-1) *(1-(1-\exp (-$

$\left.\hookrightarrow \quad 1 * \mathrm{x}))^{\wedge} \mathrm{g}\right)^{\wedge}(\mathrm{b}-1)$

\}

cdf $3<-$ function(params, $x$ )

\{

$\mathrm{g}<-$ params $[1]$

$\mathrm{m}<-$ params $[2]$

$\mathrm{b}<-1$

$1<-$ params [3]

$\operatorname{Ibeta}\left((1-\exp (-1 * \mathrm{x}))^{\wedge} \mathrm{g}, \mathrm{m}, \mathrm{b}\right)$

\}

result3 $=$ goodness.fit $(\operatorname{pdf}=$ pdf $3, \mathrm{cdf}=\mathrm{cdf} 3$, starts $=c(1,1,1)$, data $=$ wind, method $=$ "BFGS", domain $=c(0$, Inf $), \mathrm{mle}=$ NULL, lim_inf $=c(0,0,0)$,

$\lim _{-}$sup $=c(100,100,100), S=250$, prop=0.1, N=50)

result3

\#BE

\#negative loglikelihood function to estimate the parameters for CTTIG pdf $4<-$ function (params, $x$ )

\{ 


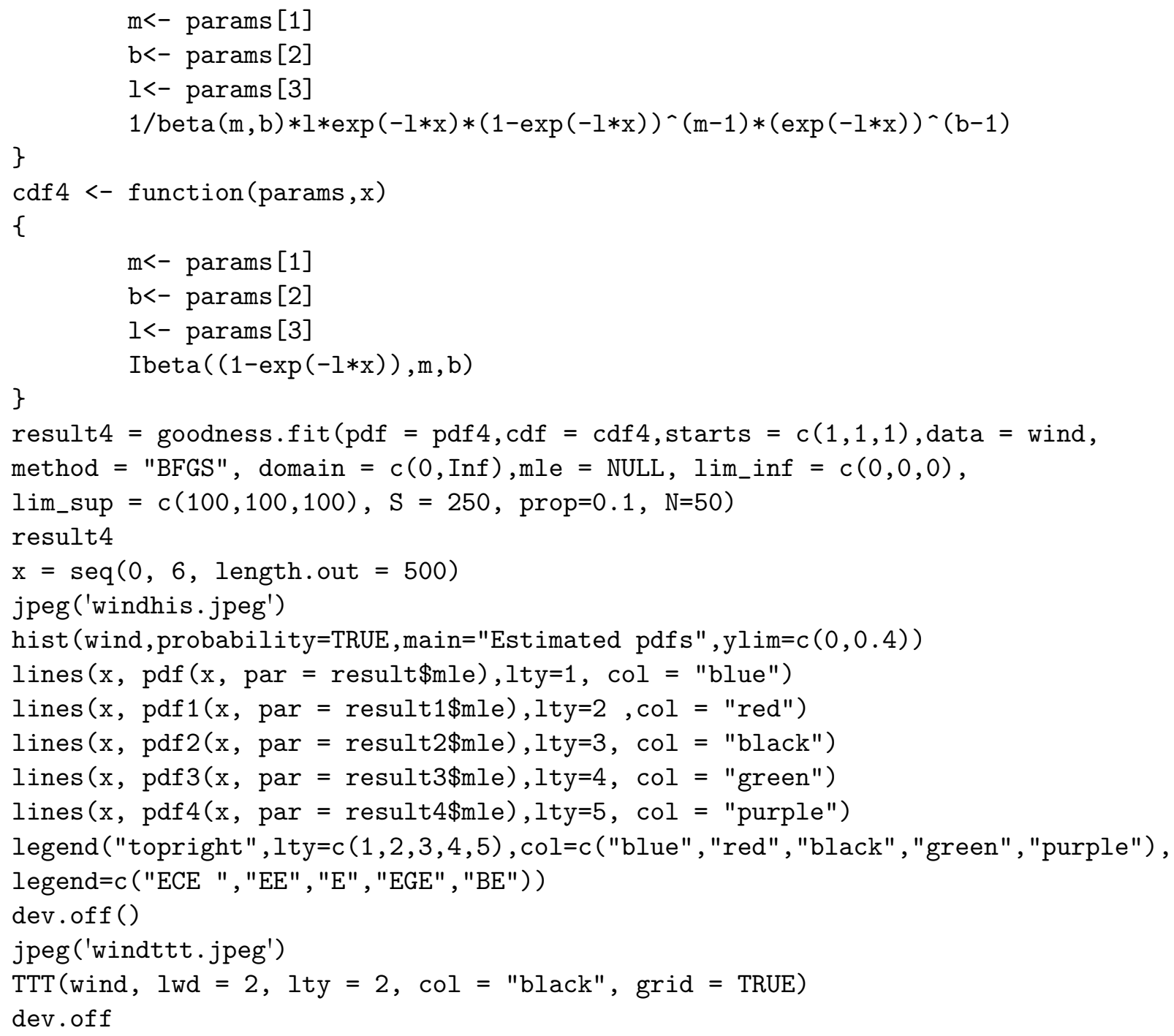

\section{Affiliation:}

Phillip Oluwatobi Awodutire

Department of Mathematical Sciences

University of Africa, Toru Orua

Bayelsa State, Nigeria

E-mail: phillip.awodutire@uat.edu.ng

\section{Austrian Journal of Statistics}

published by the Austrian Society of Statistics

Volume 51

January 2022 http://www.ajs.or.at/

http://www.osg.or.at/

Submitted: 2020-10-19

Accepted: 2021-05-24 